\title{
Lysyl-tRNA synthetase-expressing colon spheroids induce M2 macrophage polarization to promote metastasis
}

\author{
Seo Hee Nam, ${ }^{1,2}$ Doyeun Kim, ${ }^{3}$ Doohyung Lee, ${ }^{2}$ Hye-Mi Lee, ${ }^{4}$ Dae-Ceun Song,,${ }^{2,5}$ Jae Woo Jung, ${ }^{1}$ Ji Eon Kim, ${ }^{2}$ Hye-Jin Kim, ${ }^{2}$ \\ Nam Hoon Kwon, ${ }^{3}$ Eun-Kyeong Jo, ${ }^{4}$ Sunghoon Kim, ${ }^{1,2,3}$ and Jung Weon Lee ${ }^{1,2,3}$ \\ IInterdisciplinary Program in Cenetic Engineering, ${ }^{2}$ Department of Pharmacy, Research Institute of Pharmaceutical Sciences, College of Pharmacy, and ${ }^{3}$ Medicinal Bioconvergence Research Center, Seoul \\ National University, Seoul, Republic of Korea. ${ }^{4}$ Department of Microbiology, Infection Control Convergence Research Center, Chungnam National University School of Medicine, Daejeon, Republic of Korea. \\ ${ }^{5}$ Systems Biotechnology Research Center, Korea Institute of Science and Technology (KIST), Gangneung-si, Gangwon-do, Republic of Korea.
}

\begin{abstract}
Lysyl-tRNA synthetase (KRS) functions canonically in cytosolic translational processes. However, KRS is highly expressed in colon cancer, and localizes to distinct cellular compartments upon phosphorylations (i.e., the plasma membranes after T52 phosphorylation and the nucleus after $\mathbf{S 2 0 7}$ phosphorylation), leading to probably alternative noncanonical functions. It is unknown how other subcellular KRSs crosstalk with environmental cues during cancer progression. Here, we demonstrate that the KRS-dependent metastatic behavior of colon cancer spheroids within 3D gels requires communication between cellular molecules and extracellular soluble factors and neighboring cells. Membranous KRS and nuclear KRS were found to participate in invasive cell dissemination of colon cancer spheroids in 3D gels. Cancer spheroids secreted CAS6 via a KRSdependent mechanism and caused the $\mathrm{M} 2$ polarization of macrophages, which activated the neighboring cells via secretion of FGF2/CRO $\alpha / \mathrm{M}$-CSF to promote cancer dissemination under environmental remodeling via fibroblast-mediated laminin production. Analyses of tissues from clinical colon cancer patients and $\mathrm{Krs}^{-/+}$animal models for cancer metastasis supported the roles of KRS, GAS6, and M2 macrophages in KRS-dependent positive feedback between tumors and environmental factors. Altogether, KRS in colon cancer cells remodels the microenvironment to promote metastasis, which can thus be therapeutically targeted at these bidirectional KRS-dependent communications of cancer spheroids with environmental cues.
\end{abstract}

\section{Introduction}

Dissemination of cells from the primary tumor can lead to metastasis to distal tissues. Disseminated cancer cells migrate and invade the stroma to reach vessels or lymph nodes $(1,2)$. Among cells within the primary tumor, metastatic cells are heterogeneous and have differential metastatic potential. Metastatic cells express factors that are able to interact with environmental cues (3). Some of these metastatic factors allow crosstalk with intracellular and extracellular factors to ultimately facilitate successful metastasis (4). The identification and subsequent inhibition of these metastatic factors would be clinically beneficial for cancer treatment.

Factors that facilitate cancer metastasis have been widely investigated. Cytosolic aminoacyl-tRNA synthetases (AARSs) participate in tumorigenesis and metastasis, even though they are typically involved in protein translation (5). Of the AARSs, lysyl-tRNA synthetase (LysRS, or KRS) is highly expressed in colon cancer cells (6) and has noncanonical functions in immune response $(7)$ and tumor metastasis $(8,9)$. KRS appears to be phosphorylated at multiple residues, leading to release from the cyto-

Conflict of interest: The authors have declared that no conflict of interest exists. License: Copyright 2018, American Society for Clinical Investigation.

Submitted: January 12, 2018; Accepted: August 28, 2018

Reference information: J Clin Invest. 2018;128(11):5034-5055.

https://doi.org/10.1172/JCI99806. solic multi-tRNA synthetase complex (MSC). Phosphorylation of S207 by ERKs results in translocation to the nucleus and is associated with improved mean disease-free survival in patients with EGFR mutations (10). Nuclear KRS enhances diadenosine tetraphosphate $\left(\mathrm{Ap}_{4} \mathrm{~A}\right)$ levels to regulate microphthalmiaassociated transcription factor-mediated (MiTF-mediated) gene expression in immunologically activated mast cells (7). S207phosphorylated KRS is also correlated with enhanced HIV infection (11). Moreover, phosphorylation of the T52 residue of KRS by $\mathrm{p} 38$ MAPK causes KRS to dissociate from the MSC and translocate to the plasma membrane. At the plasma membrane, KRS associates with and stabilizes 67-kDa laminin receptor (p67LR) and $\alpha_{6} \beta_{1}$ integrin, promoting an intracellular signal for migration $(6,12)$. The protein-protein interaction between p67LR and KRS has been the target of anti-KRS reagents to inhibit KRS-mediated cell migration and metastasis (9). It was recently reported that cleavage of the $\mathrm{N}$-terminus of KRS by caspase- 8 enables syntenin to bind to truncated KRS before secretion of KRS-containing exosomes from colon cancer cells, which attracts macrophages that cause inflammation (13). However, these studies did not address the bidirectional communication between KRSpositive cancer cells and microenvironmental factors. Importantly, the significance of KRS in the communications necessary for metastasis has not been explored in 3D, extracellular matrixsurrounded (ECM-surrounded) coculture conditions. 
The tumor microenvironment plays a pivotal role in cancer progression and metastasis. The microenvironmental components involved in tumor progression and metastasis are diverse and include the ECM; neighboring cells, such as tumor-associated macrophages (TAMs), cancer-associated fibroblasts (CAFs) (14), and endothelial cells; and soluble factors such as cytokines, chemokines, and growth factors (15). The bidirectional communication between cancer cells and their microenvironment is variable because of the heterogeneity and transformation potential of the cancer cells and the dynamic composition and activity of the microenvironment (16). Macrophages respond differently to a variety of microenvironmental signals that originate from cancer cells and can therefore have antitumorigenic or protumorigenic functions (17). Macrophages undergo substantial phenotypic changes in response to their diverse interactions with tumor cells that lead to 2 possible phenotypes: M1 (classically activated) macrophages, which produce proinflammatory cytokines that strongly inhibit pathogens and tumor cells, and M2 (alternatively activated) macrophages, which produce antiinflammatory cytokines (18). In malignant tumors, TAMs primarily resemble the M2 phenotype, although their exact phenotype and characteristics are still unclear. TAMs promote cancer progression and metastasis by secreting a variety of cytokines. TAM-derived cytokines can promote cancer cell migration and invasion by modulating cell-ECM adhesions in the tumor microenvironment (3). In addition to TAMs, CAFs contribute to cancer metastasis by producing ECM components that lead to stromal remodeling and increase the migratory and invasive capacities of the tumor (19). The significance of the communication between KRS in cancer cells and environmental factors in 2D or 3D culture systems is unknown.

In this study, we investigated how KRS in colon cancer spheroids embedded in 3D collagen I gels communicated with environmental cues, including neighboring cells and soluble factors, to promote metastasis. The KRS-dependent molecular profiles for intracellular communication were further studied in human and animal colon cancer tissues. We found that KRS expression in colon cancer cells caused M2 polarization of macrophages, which then secreted cytokines and chemokines that activated cancer cells and CAFs. The activation of CAFs induced laminin expression, leading to tissue remodeling at the cancer-stromal interface. Importantly, KRS-positive cancer cells, unlike KRS-negative cells, had a close relationship with M2 macrophages and CAFs during the remodeling of the tumor environment. Therefore, KRS is a promising target for the development of therapeutic reagents against cancer metastasis.

\section{Results}

Macrophages promote KRS-dependent cell dissemination from colon cancer spheroids embedded in 3D collagen I gels. Public Oncomine data revealed that KRS is overexpressed in colon cancer patients, compared with the normal control group (Figure 1A). We have previously reported that KRS-positive colon cancer cells play roles in cancer metastasis (9), and colon cancer spheroids in 3D collagen I gels disseminate or exhibit invasive outgrowths via KRS-dependent ERKs and paxillin activity (12). However, the influence of the environment on the ERKs and paxillin activitydependent dissemination from 3D tumor masses during KRS- dependent colon cancer metastasis has not been explored. To determine the effects of environmental factors on metastatic cell migration outbound from the tumor masses, we used conditioned media (CM) from macrophages and THP-1 cells or primary human monocytes. HCT116 or SW620 colon cancer cells were processed to form spheroids using stably transfected control cells with control shRNA (shControl), KRS-suppressed cells with shRNA against KRS (shKRS\#2 or shKRS\#5), or KRSoverexpressing cells with KRS-WT plasmids. The KRS expression levels in the stable cell lines were correlated to ERK activity (Figure 1B), as previously described (12). The embedded spheroids were treated with control media or CM from THP-1 M1 or M2 macrophages, and time-lapse images were captured for 1 day (1:00:00) or 2 days (2:00:00). Compared with the control spheroids (i.e., shControl spheroids with endogenous KRS expression), treatment with CM from either the THP-1 M1 or M2 macrophages enhanced the disseminative phenotypes (Supplemental Figure 1A; supplemental material available online with this article; https://doi.org/10.1172/JCI99806DS1). In KRS-suppressed spheroids, the control media treatment did not induce effective dissemination; however, treatment with CM from THP-1 M1 or M2 macrophages recovered the phenotype (Supplemental Figure 1A). In addition, direct treatment of the soluble cytokines that were used in the differentiation of macrophages did not cause invasive outgrowth of the colon cancer spheroids (Supplemental Figure 1B), suggesting that components secreted by the M2 macrophages were responsible for the effects. The phenotypes in 3D gels positively correlated with the activation of ERKs and STAT3 and the expression and activation of paxillin (Figure 1C). Without the CM treatment, shControl colon cancer spheroids showed basal levels of invasive outgrowth or dissemination.

Treatment with CM from human M1 or M2 macrophages promoted the dissemination of shControl and even KRS-suppressed spheroids, although there were slight differences in the effect of CM from M2 and M1 macrophages (Figure 1D). Specifically, human $\mathrm{M} 2$ macrophage-CM was superior to M1 macrophage-CM in causing invasive cell migration and increasing STAT3 and ERK signaling activation (Figure $1 \mathrm{E}$ ). The effects of $\mathrm{M} 2$ macrophage-CM on the outgrowth of colon cancer spheroids in 3D correlated with the activation of FGFR, STAT3, p38, paxillin, and ERKs (Figure 1, C and E, and Supplemental Figure 2, A and B). Interestingly, inhibition of ERKs abolished STAT3 activity (Supplemental Figure 2C). Notably, the difference in cell dissemination caused by the M1 macrophage-CM from THP-1 cells versus human monocytes might be due to the fact that THP- 1 cells are a human monocytic cell line derived from an acute monocytic leukemia patient and may differ from primary monocytes from healthy individuals.

Soluble factors produced by M2 macrophages cause membranous KRS-positive cancer cells to disseminate. To determine which soluble factors in the CM from THP- 1 cells and human primary monocytes and macrophages were important for the promotion of cell outgrowth, we performed antibody array analyses. We found that FGF2, growth-regulated oncogene- $\alpha$ (GRO $\alpha)$, macrophage colony-stimulating factor (M-CSF), osteopontin, and serpin E1 were more commonly found in the M2 macrophage-CM than in the CM from monocytes (THP-1 cells or human primary) or M1 
A

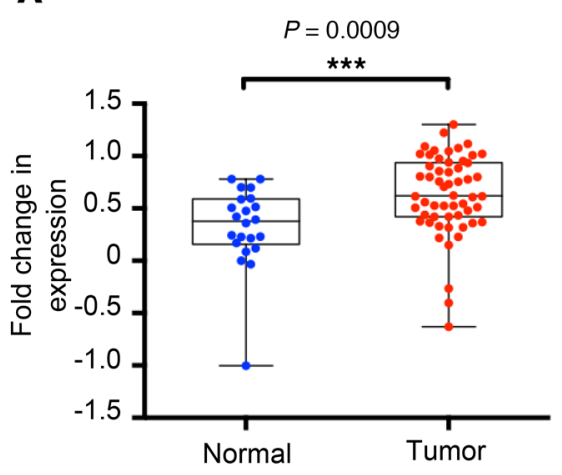

B

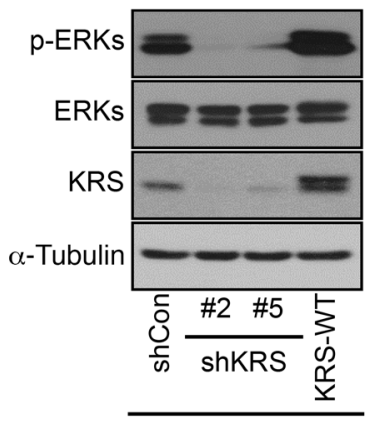

HCT116 in 3D collagen
D

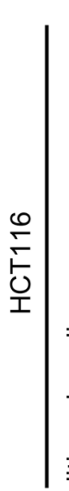

$\mid$
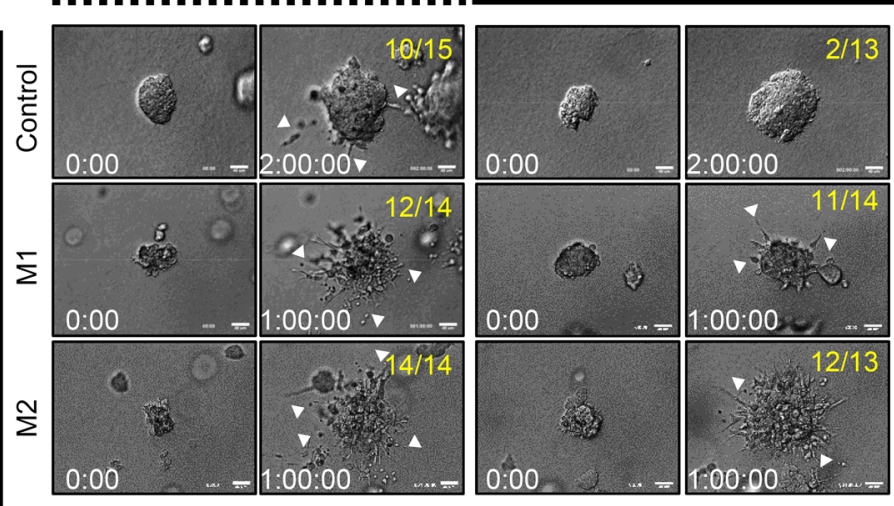

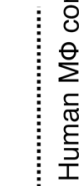

莡
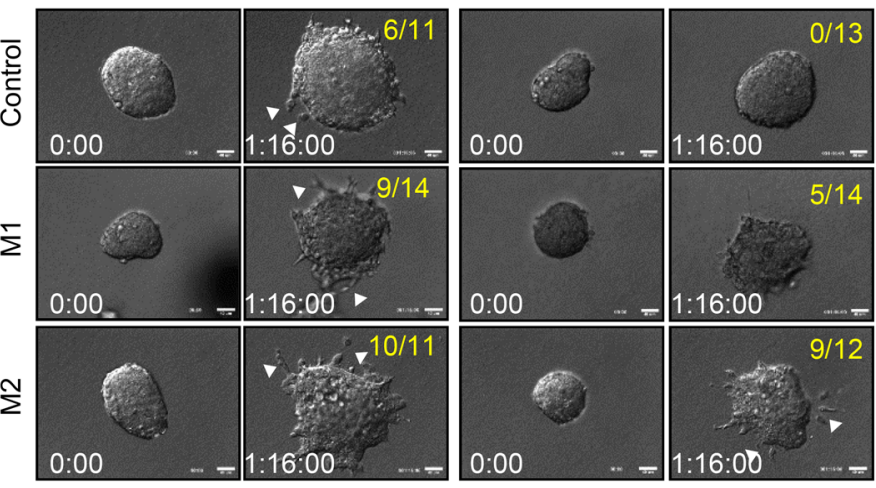

C

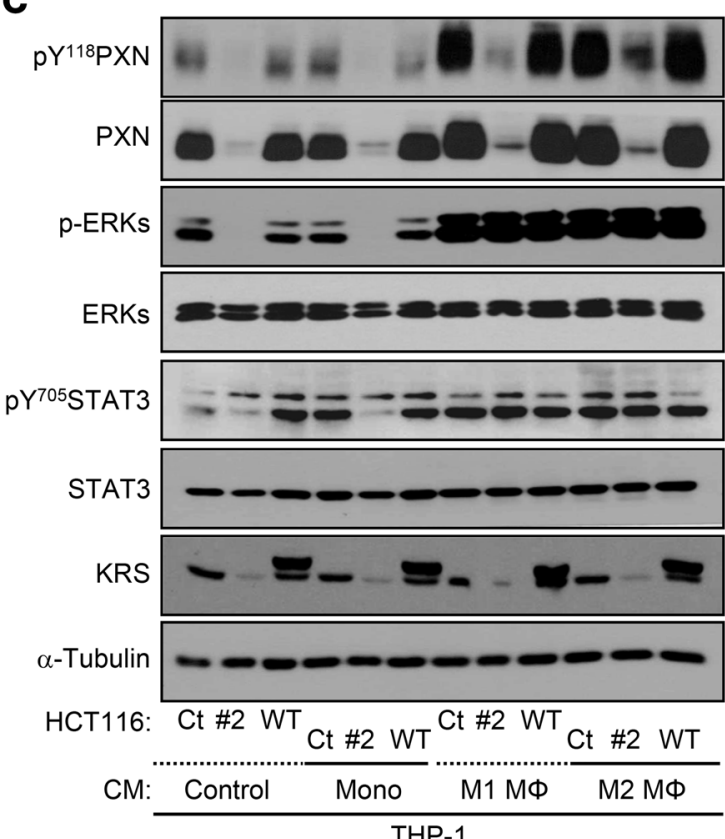

E

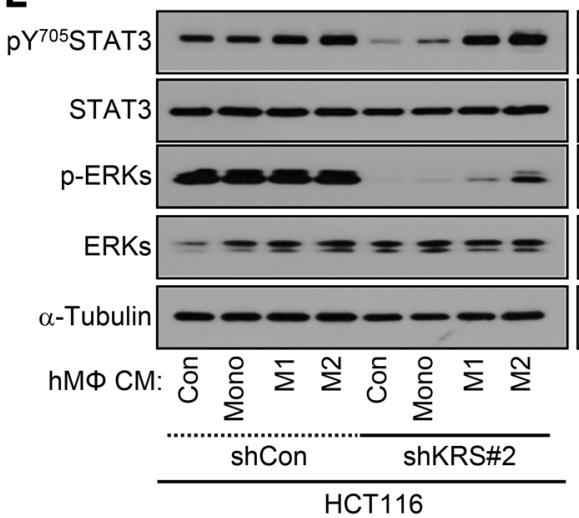

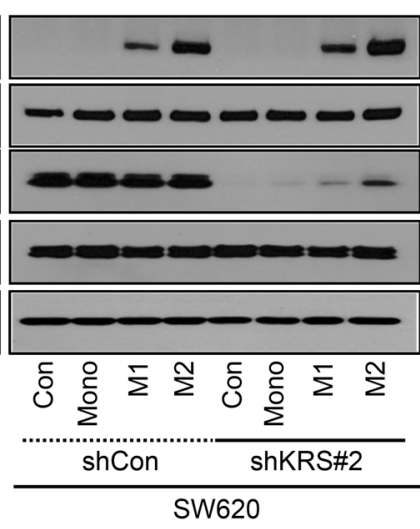

Figure 1. Cell dissemination from KRS-positive colon cancer spheroids embedded in $3 \mathrm{D}$ collagen I gels is promoted by the conditioned media of $\mathrm{M} 2$ macrophages. (A) Public Oncomine data analysis showed overexpression of KRS in colon cancer patients. The data are presented as the mean \pm SD. ${ }^{* *} P<0.001$ by Student's $t$ test. (B) Colon cancer HCT116 spheroids (using shControl, KRS-suppressed shKRS\#2 or shKRS\#5, and KRS-WT-overexpressing stable cells) in 3D collagen I gels were analyzed by standard Western blots. (C-E) HCT116 or SW620 spheroids in 3D collagen I gels were time-lapse-imaged for the indicated periods $\left(0^{\mathrm{d}}: 00^{\mathrm{h}}: 00^{\mathrm{min}}\right)$ after treatment with conditioned media (CM) of THP-1 (C) or normal human monocytes and differentiated macrophages ( $\mathbf{D}$ and $\mathbf{E})$. After imaging, whole extracts prepared from the spheroids were normalized and immunoblotted ( $\mathbf{C}$ and $\mathbf{E}$ ). Spheroid images include yellow fractions to depict the phenotypes observed (numerator) out of the total spheroids (denominator) analyzed (D). Scale bars: $40 \mu \mathrm{m}$. The data shown represent 3 independent experiments. See also Supplemental Figures 1 and 2. 
A

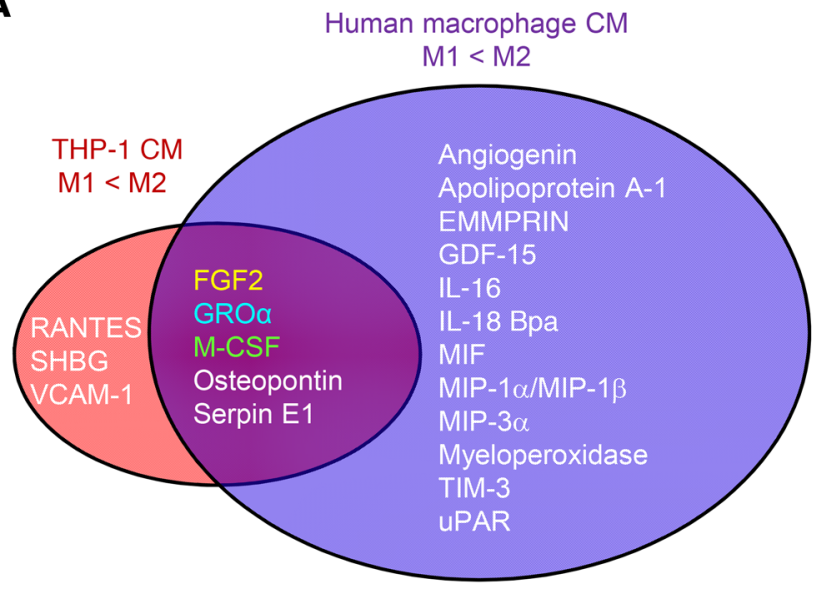

B

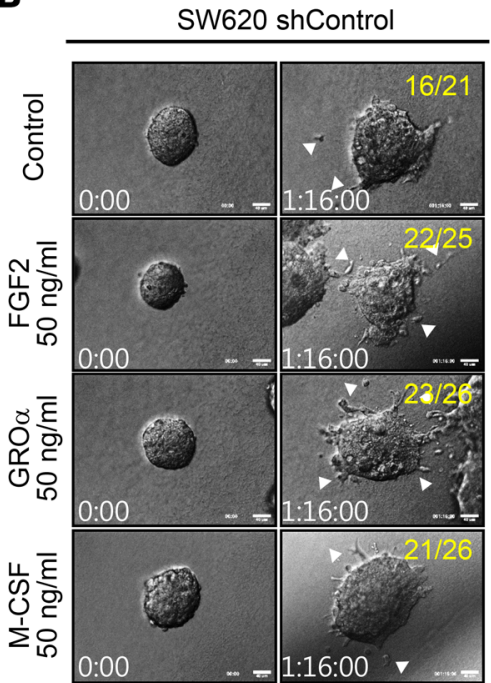

SW620 ShKRS\#2

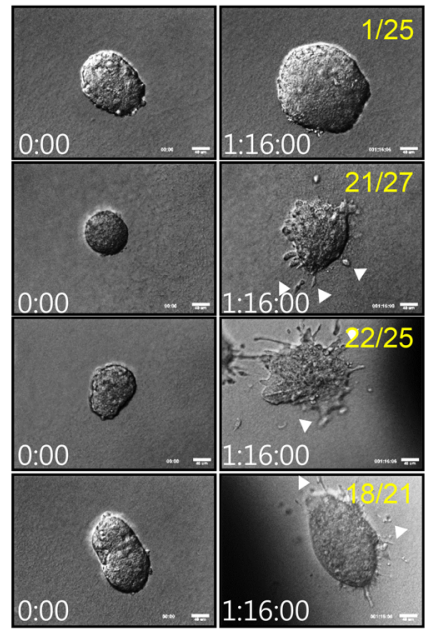

C

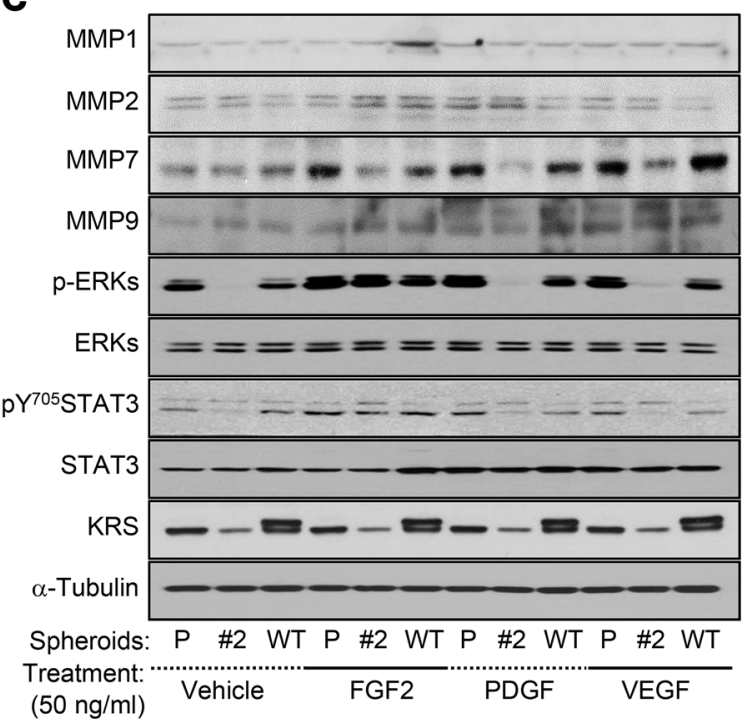

D

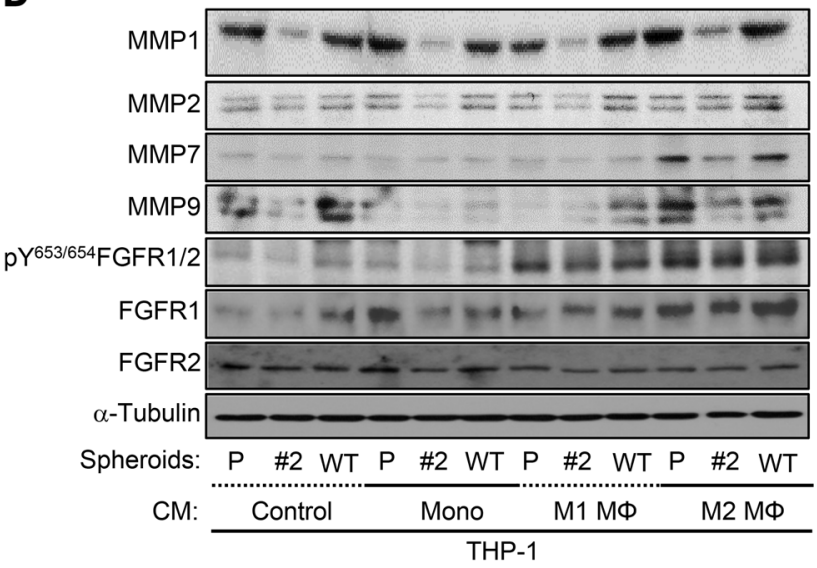

E

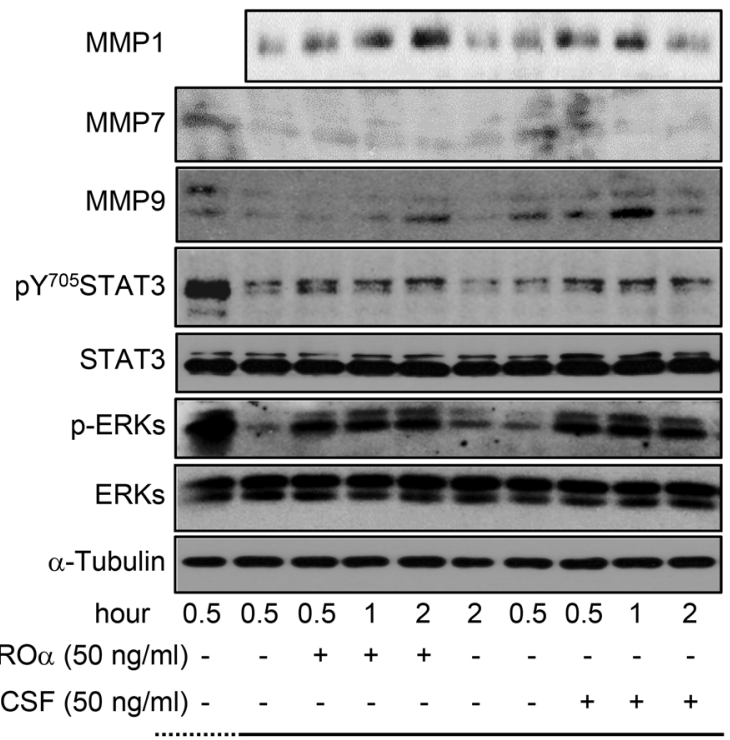

Spheroids: $\mathrm{P}$

macrophages (Figure 2A and Supplemental Figure 3, A and B). Because osteopontin and serpin E1 levels in the CM were low, we focused on the effects of FGF2, GRO $\alpha$, and M-CSF on invasive cell migration. M1 macrophages exhibited elevated mRNA
Figure 2. M2 macrophage-produced cytokines promote signaling activities for disseminative outgrowths from membranous KRS-positive spheroids in 3D gels. (A) Diagram indicating cytokine production by macrophages differentiated from THP-1 cells. (B) Vehicle (control) or cytokines that were shown to be produced preferentially by M2 macrophages were administered to KRS-positive and KRS-suppressed spheroids embedded in 3D collagen I gels, before time-lapse imaging for 40 hours $\left(1^{\mathrm{d}}: 16^{\mathrm{h}}: 00^{\mathrm{min}}\right)$. Snap images for the starting and ending points are presented. Spheroid images include yellow fractions to depict the phenotypes (numerator) out of total spheroids (denominator) analyzed. Scale bars: $40 \mu \mathrm{m}$. (C-E) HCT116-shControl (P), HCT116-shKRS\#2 (\#2), or HCT116-KRS-WT (WT) spheroids were treated with cytokines (C); CM from control, THP-1 monocytes, THP-1 differentiated M1 macrophages, or THP-1 differentiated M2 macrophages (D); or GRO $\alpha$ or M-CSF (E), as explained in Methods. One day after, whole cell extracts were prepared for immunoblotting. The data shown represent 3 independent experiments. See also Supplemental Figure 3. levels of FGF2, GROA, and MCSF upon treatment with CM from KRS-positive spheroids, and the levels were comparable to those of the M2 macrophages (Supplemental Figure 3B). Treatment with each cytokine alone promoted invasive outgrowth 
A
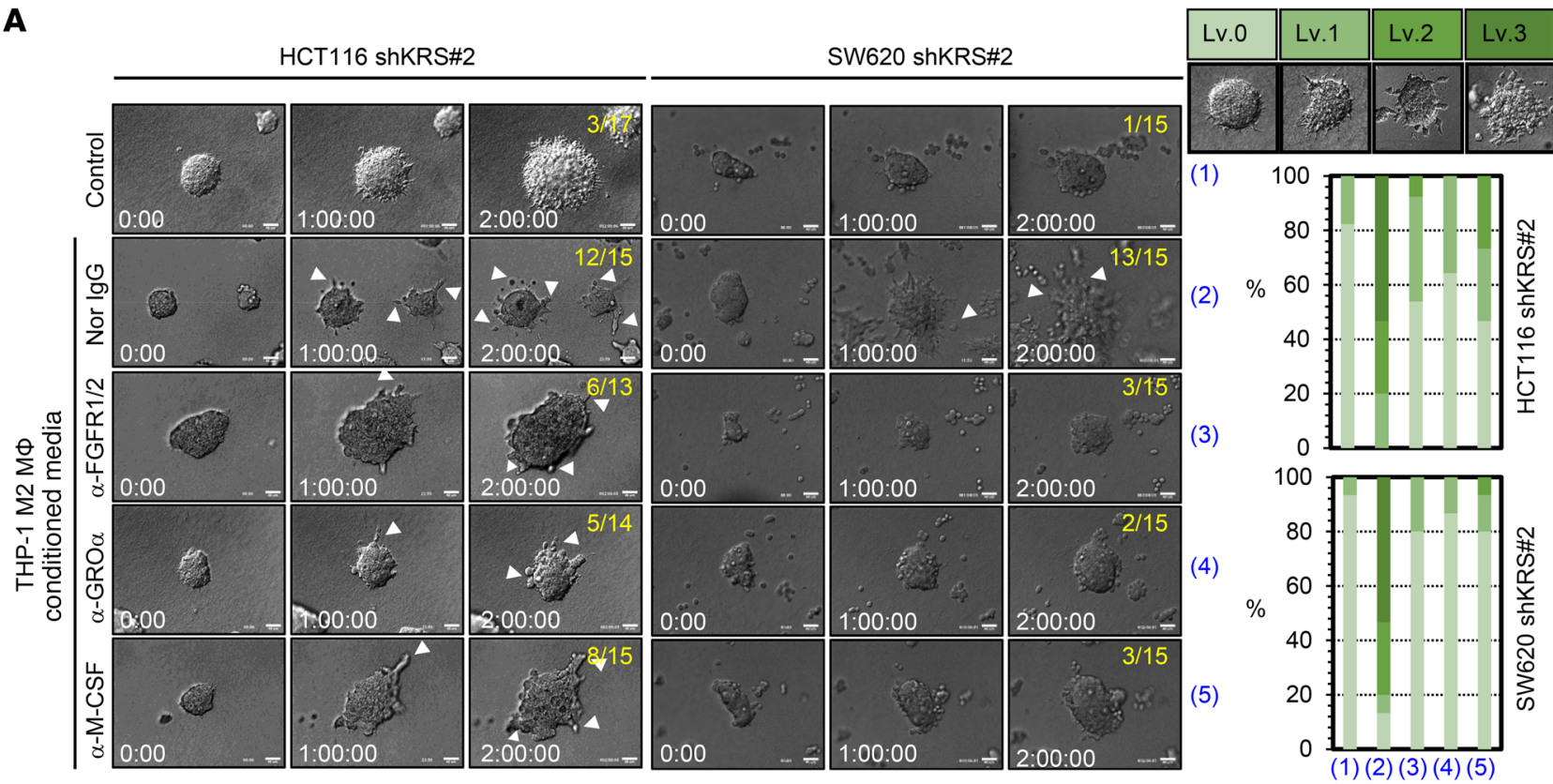

B

HCT116 shContro

HCT116 KRS-WT

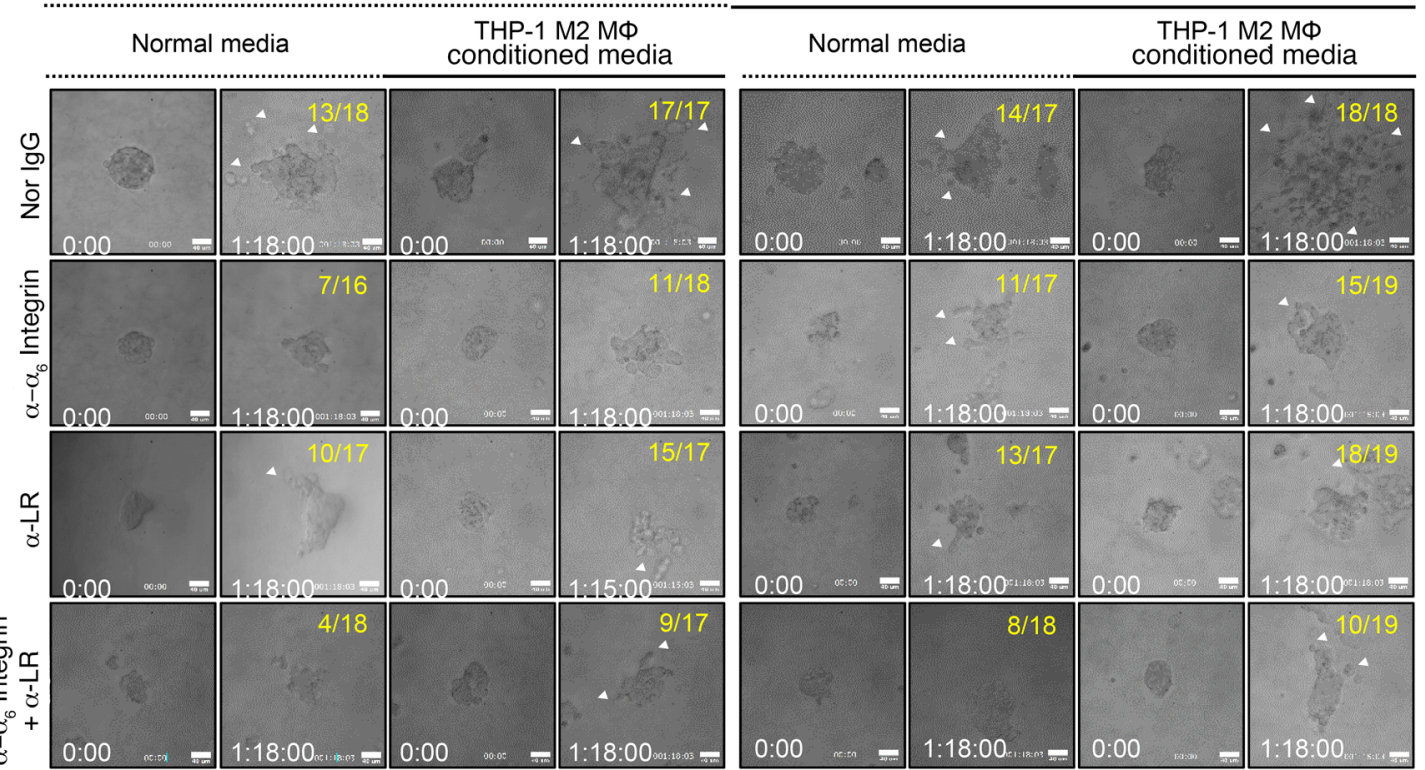

C

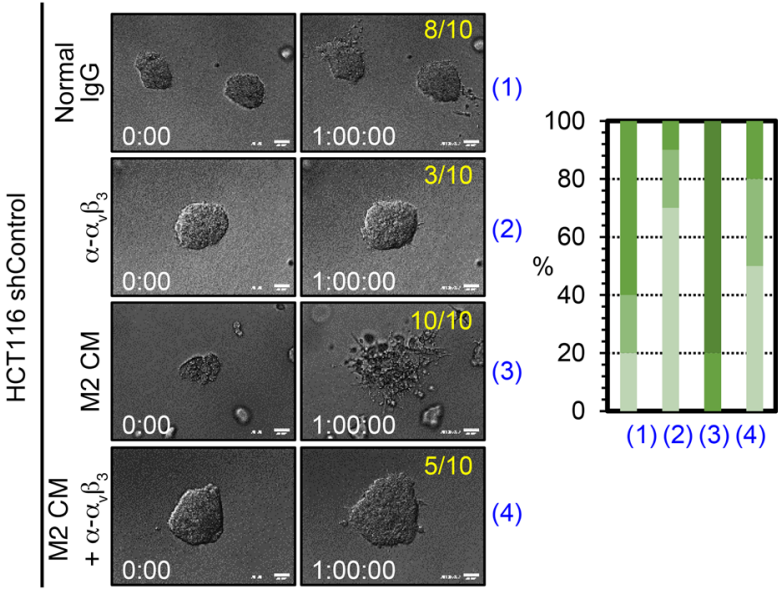

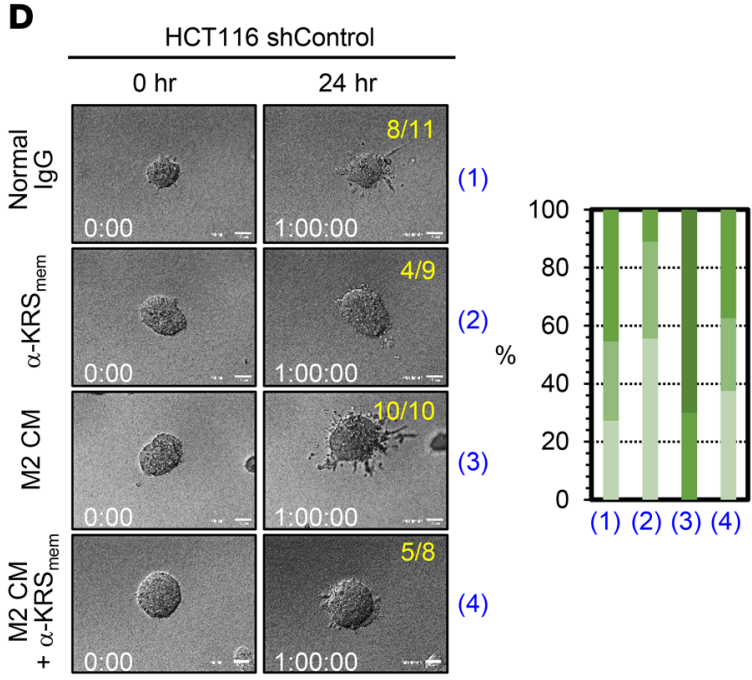


Figure 3. Roles of membranous KRS in the disseminative outgrowths from 3D spheroids. (A) KRS-suppressed colon cancer spheroids (shKRS\#2) were treated with $\mathrm{M} 2$ macrophage-CM for 2 days $\left(2^{\mathrm{d}}: 00^{\mathrm{h}}: 00^{\mathrm{min}}\right)$ in the absence or presence of antibodies against FGFR, GRO $\alpha$, or M-CSF and were time-lapse-imaged. (B) Cells were embedded in 3D collagen I gels with normal media or M2 macrophage-CM. In addition, the 3D culture medium was treated with normal IgG (Nor IgC) or antibodies to neutralize human $\alpha_{6}$ integrin, p67LR, or both, before time-lapse imaging for 1 day and 18 hours ( $\left(1^{\mathrm{d}}: 18^{\mathrm{h}}: 00^{\mathrm{min}}\right)$. (C and $\left.\mathbf{D}\right)$ KRS-positive spheroids (shControl) were treated with normal IgC or antibodies against $\alpha_{v} \beta_{3}$ integrin (C) or membranous KRS (KRS ${ }_{\text {mem }}$; D) with or without concomitant treatment of M2 macrophage-CM. The disseminative phenotypes (shown in $\mathbf{A}$ ) for each experimental condition are also presented in graphs as percentage values (A, C, and D). The data shown represent 3 independent experiments. See also Supplemental Figure 4. Scale bars: $40 \mu \mathrm{m}$.

of the shControl colon cancer spheroids, and the disseminative phenotype was recovered in the KRS-suppressed spheroids (Figure 2B). Unlike other growth factors, such as PDGF and VEGF, FGF2 promoted the activity of STAT3 and ERKs in HCT116-shKRS\#2 spheroids to the levels of KRS-positive spheroids (Figure 2C and Supplemental Figure 3C). Furthermore, treatment of the HCT116 spheroids with M1 or M2 macrophage-CM caused FGFR1/2 activation and FGFR1 expression in KRS-positive (i.e., shControl and KRS-overexpressing) and KRS-suppressed spheroids, whereas treatment with monocyte $\mathrm{CM}$ showed results similar to those in the control media-treated spheroids (Figure 2D). Interestingly, M2 macrophage-CM also slightly promoted expression of matrix metalloproteinases (MMPs), including MMPs 1, 2, 7, and 9 (Figure 2, C and D). The stimulatory effects of GRO $\alpha$ and M-CSF on the activation of STAT3 and ERKs in KRS-positive shControl-expressing and KRS-suppressed (shKRS\#2) spheroids were obvious after treatments for less than 2 hours (Figure 2E). In contrast, the effects of FGF2 were notable after 12 (unpublished observations) or 24 hours of treatment (Figure $2 \mathrm{C}$ and Supplemental Figure 3C), indicating that the roles of FGF2, GRO $\alpha$, and M-CSF in cell dissemination might be mechanistically different. MMP1 and MMP9 were also obviously upregulated by FGF2, GRO $\alpha$, and M-CSF (Figure 2, $\mathrm{C}$ and $\mathrm{E}$ ).

To confirm that the soluble factors were important for the observed effects, neutralization by antibody incubation was performed during time-lapse imaging of the invasive migration promoted by the CM from THP-1 M2 macrophages. Whereas normal IgG treatment did not affect the dissemination promoted by the $\mathrm{CM}$ from M2 macrophages, neutralization using anti-FGFR1/2, anti$\mathrm{GRO} \alpha$, or anti-M-CSF antibodies partially abolished the outgrowth of KRS-suppressed colon spheroids that were recovered by the M2 macrophage-CM treatment (Figure 3A). In KRS-positive spheroids, invasive migration involves a protein complex on the cell surface consisting of KRS, $p 67 \mathrm{LR}$, and $\alpha_{6} \beta_{1}$ integrin (12). However, antibody neutralization of $\alpha_{6} \beta_{1}$ integrin and p67LR together could not completely block the M2 macrophage-CM-mediated dissemination, and single-antibody neutralization was less effective than the combined neutralization (Figure 3B). Together, these results suggest that a mechanism(s) independent of KRS, p67LR, and $\alpha_{6} \beta_{1}$ integrin may also contribute to M2 macrophage-CM-mediated dissemination.

To determine whether membranous KRS is necessary for the invasive migration of cells treated with M2 macrophage-CM, we first examined whether blocking or neutralizing the membrane proteins resulted in decreased dissemination. Because FGF2 and FGFR1/2 are known to bind $\alpha_{v} \beta_{3}$ integrin at the ECM-adhesion contact points during endothelial adhesion, proliferation, and migration (20), we investigated whether the functional neutralization of $\alpha_{\mathrm{v}} \beta_{3}$ integrin abolished the M2 macrophage-CMmediated dissemination. Treatment with anti-human $\alpha_{4} \beta_{3}$ integrin antibody reduced the basal and M2 macrophage-CM-mediated dissemination (Figure 3C). More importantly, treatment with an antibody against membranous KRS also blocked the basal and M2 macrophage-CM-mediated outgrowth of the spheroids (Figure 3D). Thus, the stimulatory effects of $\mathrm{M} 2$ macrophage-CM on KRS-dependent invasive outgrowth of the spheroids involved KRS on the membrane surface forming protein-protein complexes with integrins and FGFR1/2 to initiate intracellular signaling and crawling forces (this study and ref. 12). Interestingly, the mRNA levels of FGF2, GROA, and MCSF in the cancer spheroids did not depend on KRS expression levels or mutations (Supplemental Figure $4 \mathrm{~A}$ ), suggesting that the effects of the cytokines on invasive cancer migration were caused by macrophages.

KRS-positive cancer spheroids cause M2 polarization of macrophages. We then determined how cancer spheroids affected macrophages in a 3D environment. The mRNA levels of markers for M1 or M2 macrophages were analyzed by real-time PCR to study how CM from HCT116 cancer spheroids with different KRS expression levels affected macrophages. When CM from KRS-positive cancer spheroids (HCT116-shControl or HCT116-KRS-WT) were added to M1 macrophages, the expression of IL6, an M1 macrophage marker (21), was unaltered. However, IL1O and CD2O6, M2 macrophage markers (21), showed elevated expression (Figure 4A). CM from KRS-suppressed cancer spheroids did not increase the levels of M2 macrophage markers (Figure 4A), indicating that KRS-positive cancer spheroids caused polarization of M2 macrophages. When mRNA levels of the factors responsible for the M2 macrophage polarization were analyzed for their correlations with the KRS levels of the spheroids, only GAS6 (growth arrest-specific 6) and IL8 positively correlated with KRS levels (Figure 4B). Antibody arrays also showed that expression of IL-8 and angiogenin (ANG) were enhanced by KRS expression, although GAS6 was not included in the array (Supplemental Figure 4B). Because the role of GAS6 in M2 polarization of macrophages is controversial $(22,23)$, we examined whether IL-8 and ANG as well as GAS6 caused M1 macrophages to become M2 macrophages. GAS6 alone or together with IL-8 and/or ANG was added to THP-1 monocytes, M1 macrophages, or M2 macrophages before evaluation of M2 macrophages marker mRNA levels. After cytokine treatment, both CD206 and IL10 mRNA levels were elevated in M1 macrophages but not in monocytes or M2 macrophages (Figure 4, C and D), suggesting that the cytokines caused M1 macrophages to be polarized to M2 macrophages. Neither IL-8 nor ANG alone significantly affected the polarization of macrophages (Supplemental Figure 4C). In addition to mRNA levels, protein levels of TNF- $\alpha$ (an M1 macrophage marker) and IL-10 (an M2 macrophage marker) also revealed KRSmediated M2 polarization (Figure 4E). Additionally, protein markers of M1 macrophages (IL-6, IL-12, and IFN- $\gamma$ ) decreased when M1 macrophages were treated with CM from KRS-positive cancer spheroids, but were not changed or less decreased when M1 
A

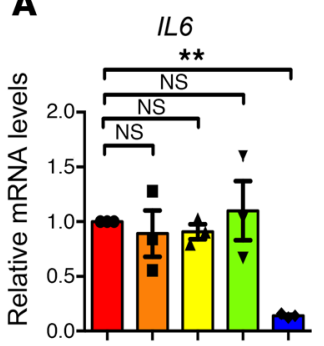

IL10 CD206
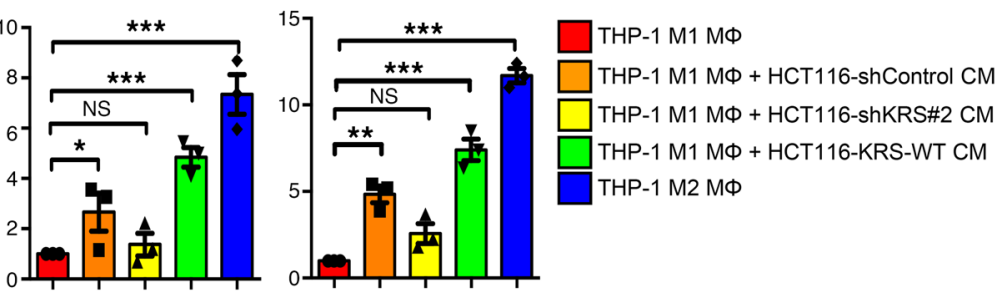

B

128
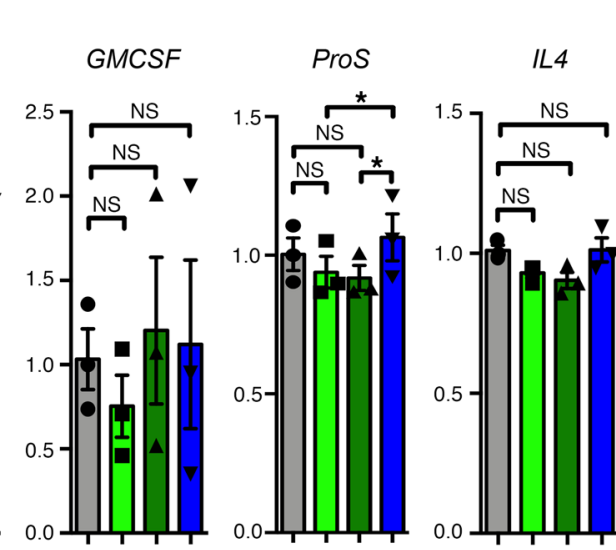

$\square$ shControl $\square$ shKRS\#2 $\square$ shKRS\#5
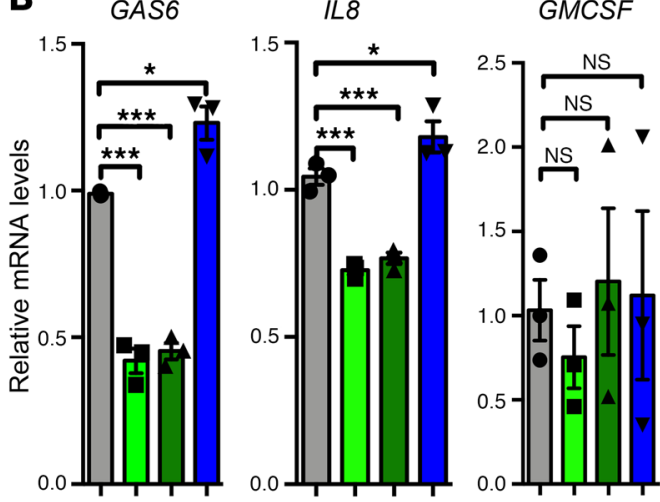

D
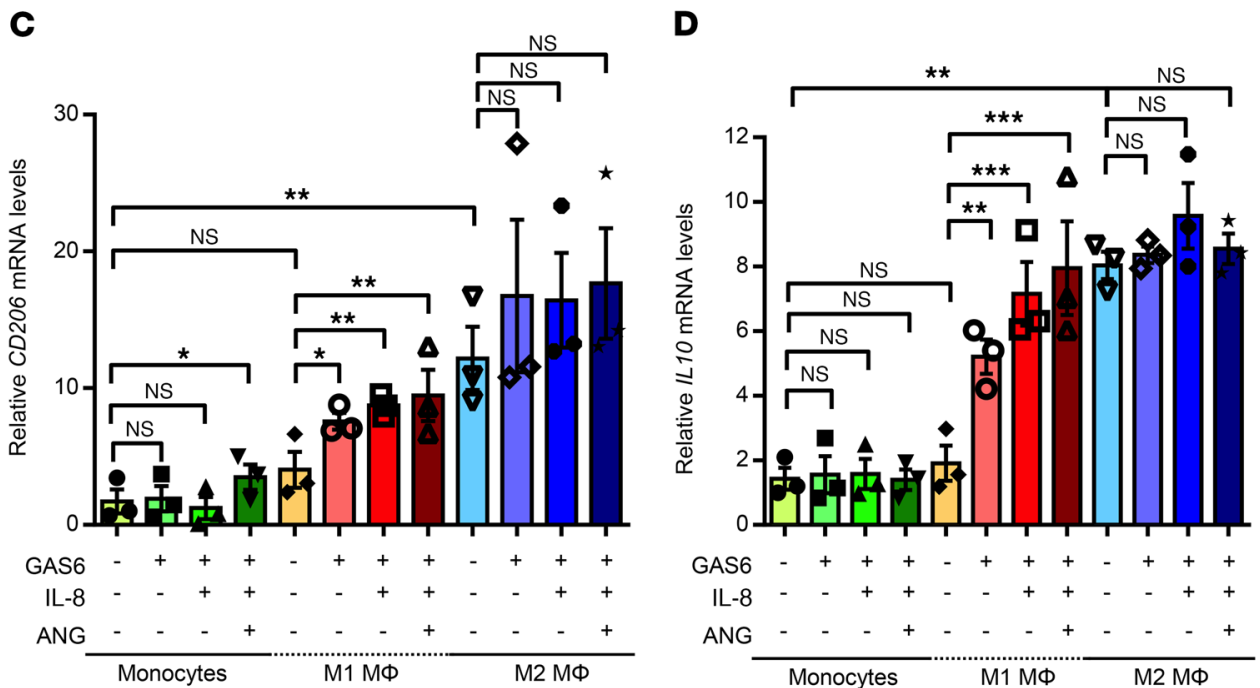

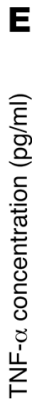
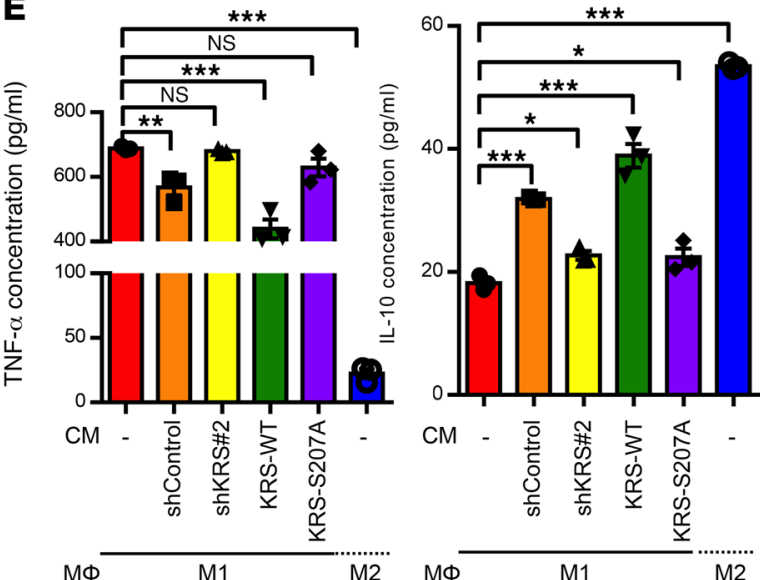

$\mathbf{F}$

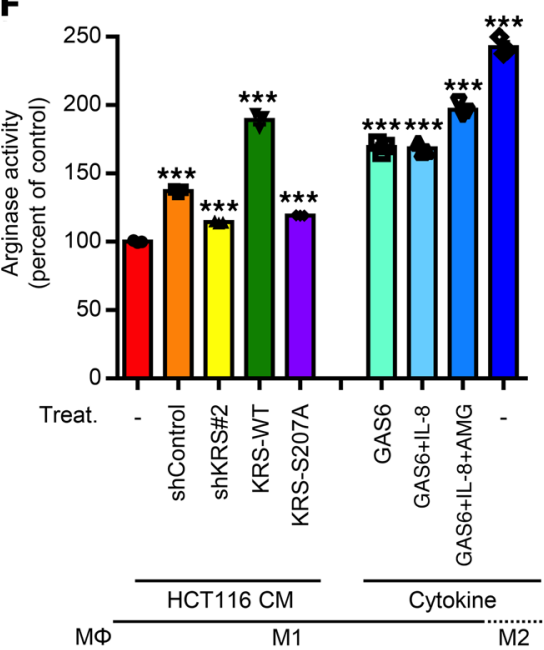

Figure 4. Nuclear KRS in colon cancer spheroids increases GAS6 expression for the M2 polarization of macrophages.

(A) The relative mRNA levels of IL6, IL10, or CD206 in THP-1 macrophages were analyzed after treatment with $\mathrm{CM}$ of HCT116 KRS-positive (shControl or KRS-WT) or KRS-suppressed (shKRS\#2) colon cancer spheroids for 24 hours. The data are presented as the mean $\pm \mathrm{SD}$. (B) GAS6, IL8, and other cytokine mRNA levels were analyzed in the $3 \mathrm{D}$ spheroids with different KRS expression levels for 24 hours. (C and D) THP-1 monocytes, M1 macrophages, or M2 macrophages were treated with GAS6, IL-8, and/or ANG, before measurement of the levels of CD206 (C) and IL10 (D) mRNA. (E and F) THP-1 M1 macrophages or M2 macrophages were treated with CM (E and F) or GAS6, IL-8, and/or ANG (F) for 24 hours before analysis of the protein levels of TNF- $\alpha$, IL-10, or arginase by ELISA. The data shown represent 3 different observations. ${ }^{*} P<0.05,{ }^{* *} P<0.01$, ${ }^{* * *} P<0.001$ by 1 -way ANOVA with Dunnett tests. See also Supplemental Figures 4 and 5. 
macrophages were treated with CM from KRS-suppressed or S207A mutant spheroids (Supplemental Figure 5). In addition, arginase activity also indicated M2 polarization of macrophages after treatment with CM from KRS-positive cells or with the KRSmediated soluble factors (GAS6, IL-8, and ANG) (Figure 4F).

M1 macrophage (CD11b) or M2 macrophage (CD206) cell surface markers were evaluated via flow cytometry using M1 macrophages treated with either $\mathrm{CM}$ from the colon cancer spheroids or cytokines. Treatment of M1 macrophages with CM from KRS-positive spheroids (shControl or KRS-WT) and/or GAS6 alone or in combination with IL-8 and/or ANG led to reduced CD11b but increased CD206 levels, indicating M2 polarization. However, treatment with CM from KRS-suppressed colon spheroids (shKRS\#2) did not cause M2 polarization (Figure 5A). In addition to the M2 macrophage markers, activation of intracellular signaling molecules such as STAT6, which is highly activated in M2 macrophages but not in M1 macrophages $(21,24)$, was also elevated by CM from HCT116 spheroids and by the cytokines (Figure 5B). Furthermore, treatment with the cytokines activated MER proto-oncogene tyrosine kinase (MerTK), a GAS6 receptor (25), in M1 macrophages (Figure 5C). The increased mRNA levels of IL1O and CD2O6 (M2 macrophage markers) after M1 macrophage treatment with $\mathrm{CM}$ from KRS-positive spheroids were reduced by additional treatment with antibodies that neutralized GAS6 or MerTK (Figure 5D). These data suggest that KRS-positive colon cancer spheroids secrete GAS6 to promote polarization of M1 macrophages into M2 macrophages.

Nuclear KRS promotes MiTF-mediated GAS6 transcription. We next determined how KRS in colon cancer spheroids led to the induction of GAS6 expression and the M2 polarization of macrophages. Fractions from HCT116 spheroids stably expressing differential levels of KRS or mutated KRS were used to confirm the cellular location of KRS. WT KRS and the $\Delta \mathrm{C} 5$ mutant, which cannot be secreted because of a 5-amino acid deletion in the C-terminus (13), were found in both the membrane and nuclear fractions. However, the T52A mutant, which cannot be dissociated from the cytosolic MSC, was located more frequently in the nucleus than in membranes, and the S207A mutant did not localize to the nucleus (Figure 6A). The $\Delta \mathrm{C} 5$ and S207A mutant spheroids still disseminated, but at lower levels than the KRS-WT spheroids, and the T52A mutant spheroids failed to disseminate (Figure 6B).

Similarly to the KRS-WT spheroids, the $\triangle \mathrm{C} 5$ and T52A mutant spheroids induced GAS6 mRNA expression, but the S207A mutant spheroids did not (Figure 6C). The ERK-mediated phosphorylation of S2O7 in KRS is important for the nuclear trafficking, leading to its involvement in MiTF-mediated transcription (26). Pharmacological inhibition of ERKs by U0126 reduced GAS6 mRNA levels in KRS-WT spheroids to a level similar to that of the S207A spheroids (Figure 6C). The insignificant GAS6 mRNA level in S207A mutant cells was linked to a reduced GAS6 protein level, whereas higher GAS6 mRNA levels in KRS-WT, $\triangle \mathrm{C} 5$ mutant, or T52A mutant cells correlated with higher GAS6 protein levels (Figure 6D). This pattern of GAS6 mRNA expression in KRS-WT or mutant spheroids correlated with the pattern of CD206 (an M2 macrophage marker) mRNA levels after M1 macrophage treatment with $\mathrm{CM}$ from the diverse KRS form-expressing spheroids (Figure 6, C and E). However, other markers for M1 macrophages, such as TNFA, ITGAX (CD11c), and IL1A, did not correlate with GAS6 mRNA levels and were inversely correlated instead (Figure 6, C and E). ELISA analysis of GAS6 protein levels revealed the comparability to GAS6 mRNA levels in diverse experimental conditions (Figure 6F): GAS6 levels were positively correlated with KRS expression, and with KRS-WT, $\Delta$ C5 mutant, and T52A mutant but not with S207A mutant expression. GAS6 levels were further enhanced by treatment of M2 macrophage-CM.

Because KRS is linked to c-Jun for paxillin transcription (12) and to MiTF for $A p 4 A$ transcription during mast cell activation (26), these transcription factors were examined using ChIP to assess KRS-activated GAS6 transcription. The regions responsive to c-Jun and MiTF were found in the promoter regions upstream of the GAS6 gene (Figure 7A). ChIP analysis using an anti-MiTF antibody showed that MiTF bound to the GAS6 promoter region 1 (R1) of KRS-positive spheroids (shControl) but not KRS-suppressed spheroids (shKRS\#2 and \#5) (Figure 7B). When CM from M2 macrophages were added to the shKRS\#2 spheroids, MiTF binding to the GAS6 promoter region 1 was recovered (Figure $7 \mathrm{~B})$. However, c-Jun was not bound to the GAS6 promoter region 2 (R2) (Figure 7B). The control region 3 (R3) of the GAS6 promoter did not recruit either MiTF or c-Jun (Figure 7B). Treatment of KRS-suppressed spheroids (shKRS\#2 or \#5) with CM from M2 macrophages resulted in enhanced KRS levels (Figure 7, C and D). The increased levels of KRS after treatment with CM from M2 macrophages involved increases in KRS mRNA levels that correlated with GAS6 transcript levels (Figure 7D). However, the expression levels of other aminoacyl-tRNA synthetases were not altered after treatment of KRS-suppressed spheroids with CM from M2 macrophages (Figure 7E). Interestingly, a small compound, YH16899, inhibited the interaction between membranous KRS and p67LR. As a result, ERK activity was negatively affected (12), but the KRSdependent GAS6 transcript level was not inhibited (Supplemental Figure 6A). FGF2, GRO $\alpha$, and M-CSF, which were effective for invasive migration of the spheroids, did not cause changes in GAS6 mRNA levels (Supplemental Figure 6B). This finding suggests that transcriptional activation of GAS6 by nuclear KRS is irrelevant to membranous KRS, which is consistent with the GAS6 mRNA levels in the T52A spheroids (Figure 6C).

Preferred recruitment of M2 macrophages by KRS-positive cancer spheroids. We then determined how KRS-positive cancer spheroids communicated with macrophages. The cancer spheroids or macrophages were labeled with fluorescent dyes, and their migration toward each other in 3D gels was analyzed using the Operetta/ Harmony High Content Screening (HCS) Platform. Red fluorescent macrophages were embedded in a monolayer at the bottom of the gel, and green fluorescent cancer cells were loaded on top of the 3D gels. Migration was monitored by the location of the red macrophages or green cancer cells in planes at 30- $\mu \mathrm{m}$ (Figure 8A) or $10-\mu \mathrm{m}$ (Figure $8 \mathrm{~B}$ ) intervals from the bottom toward the top of the gel, respectively. Compared with monocytes and M1 macrophages, more M2 macrophages migrated farther toward cancer cells, evidenced by the presence of more red cells in higher planes (i.e., planes 4-6) (Figure 8A). Interestingly, when KRS-positive cancer cells were on top of the 3D gels, the M2 macrophage populations in the higher planes were greater than when KRS-suppressed 
A

Treat. : Control
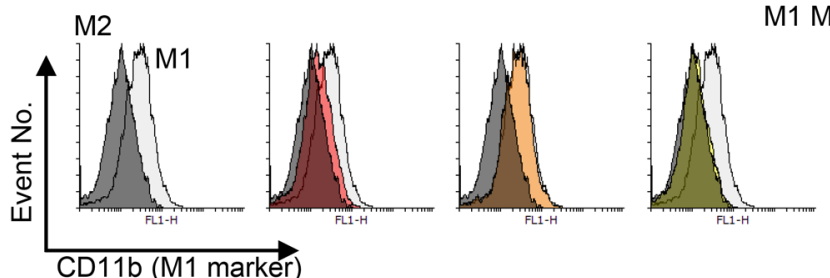

M1 MФ
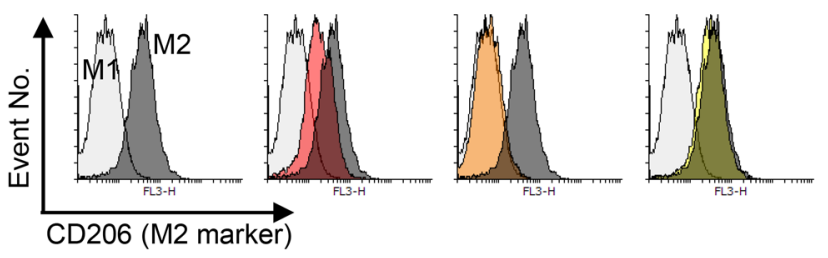

B

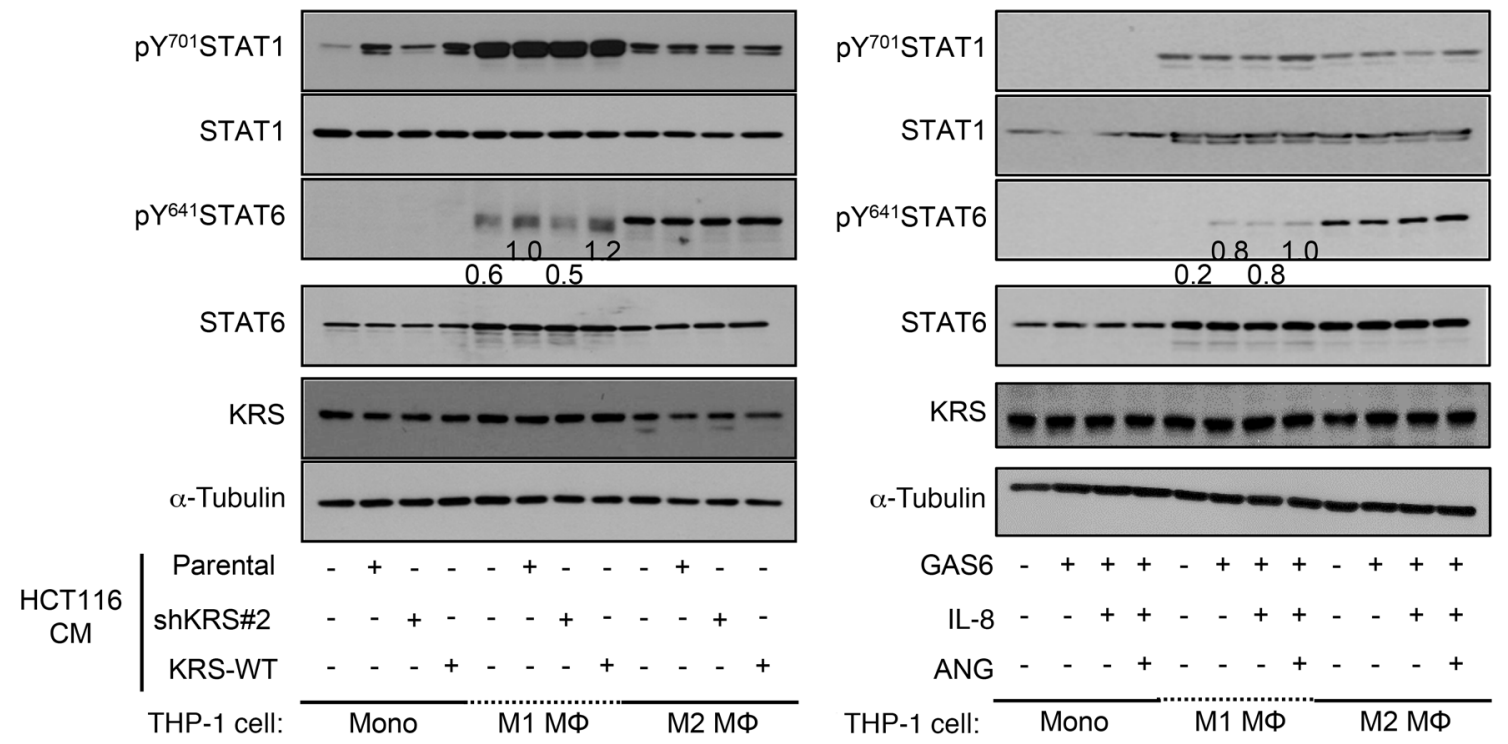

C

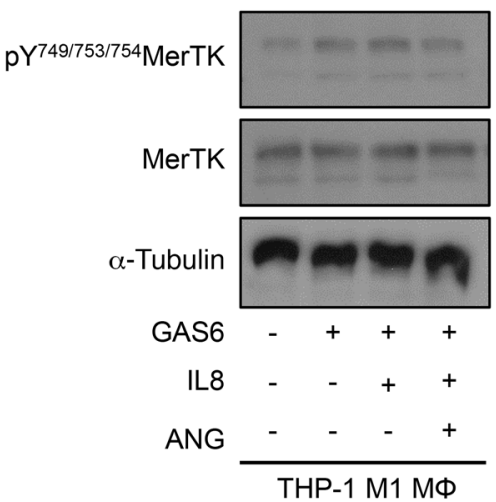

D

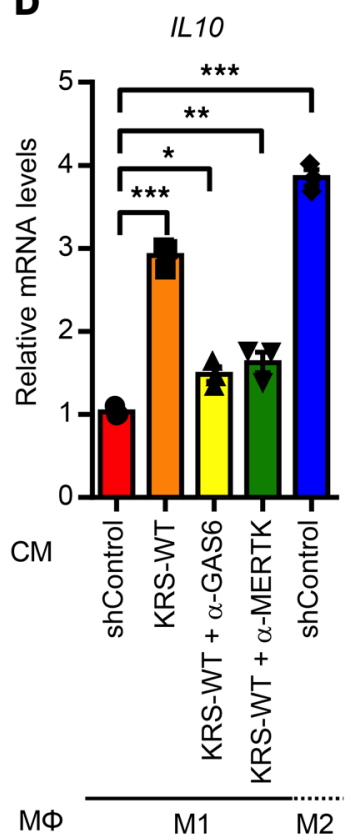
THP-1 M1 MФ
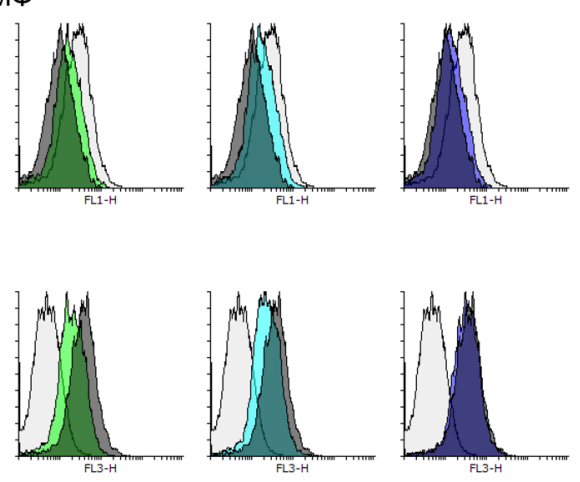

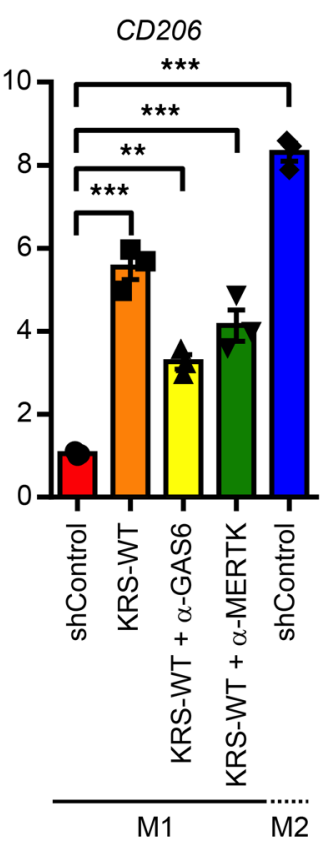


Figure 5. KRS-mediated GAS6/IL-8/ANG expression for the M2 macrophages polarization and signaling. (A and B) M1 macrophages in 3D collagen I gels were treated with CM from KRS-positive spheroids or with GAS6, IL-8, and/or ANG for 24 hours, before flow cytometry analysis of CD11b (M1 macrophage marker) and CD206 expression on the cell surface (A) or STAT6 phosphorylation, an index for M2 polarization (B). (C and D) THP-1 M1 macrophages were treated with CAS6, IL-8, and/or ANG for 24 hours, before immunoblots (C) or with the CM of KRS-WT spheroids in the absence or presence of antibodies to neutralize GAS6 and MerTK for 24 hours before IL10 and CD206 mRNA analysis (D). The data are presented as the mean $\pm \mathrm{SD} .{ }^{*} P<0.05$, ${ }^{* *} P<0.01,{ }^{* *} P<0.001$ by 1 -way ANOVA with Dunnett tests (D). The data shown represent 3 different observations

cells were on top of the gels (Figure 8A). These observations indicate that M2 macrophages migrate more efficiently toward KRSpositive cancer cells.

In the reverse experiment, green fluorescent cancer cells were seeded at the bottom of the 3D gels, and red fluorescent macrophages were layered on top. Compared with KRS-suppressed cancer cells (shKRS\#2), KRS-positive shControl cancer cells migrated to more distant planes ( $>4$ planes) and especially toward the M2 macrophage population during the 5 days of incubation (Figure 8B). When the incubation was performed for a longer period of 10 days, green-shControl cancer cells migrated similarly toward either M1 or M2 macrophages, although shKRS\#2 cells migrated slightly more toward M2 macrophages than M1 macrophages (Figure 8B). The comparable migration of shControl cancer cells to M1 or M2 macrophages may be because M1 macrophages can be polarized to M2 macrophages during an extended incubation period. To clarify the role of GAS6 in crosstalk between cancer spheroids and macrophages, we examined the migration of macrophages toward HCT116 spheroids during coculture with or without GAS6, IL-8, and/or ANG. Migration was monitored by the location of the red macrophages in planes at 10- $\mu$ m intervals from the bottom toward the top of the gel. Red fluorescent M1 macrophages that were treated for 10 days with GAS6, IL-8, and/or ANG caused migration in 3D gels toward KRS-positive shControl cells that was greater than their migration toward KRS-suppressed shKRS\#2 cells (Figure 8C). Thus, this indicates that M1 macrophages treated with soluble factors for 10 days could be polarized to M2 macrophages. Thus, GAS6 treatment clearly caused increased migration of M1 macrophages toward cancer spheroids (Figure 8C).

Next, we assessed the significance of M2 macrophages in the invasive migration of cells in 3D gels. We cocultured red shKRS\#2 or green fluorescent shControl cancer spheroids in 3D gels and captured images of their behavior at 30-minute intervals for 2 days. Spheroids mixed with KRS-suppressed shKRS\#2 and KRS-positive shControl cells showed dissemination of the green-shControl cells only, but upon treatment with CM from M2 macrophages, dissemination of even the red shKRS\#2 cells was observed (Figure 8D). Therefore, invasive outgrowth was influenced more by M2 macrophages than by the KRS-positive spheroids, suggesting that M2 macrophages can cause invasive outgrowth even of KRS-suppressed spheroids via an increase in KRS levels in the cancer spheroids (Figure 7, C-E).

The preferred recruitment of M2 macrophages by KRSpositive cancer cells was analyzed in vivo using an animal model system of colon cancer induced by azoxymethane (AOM) and dextran sodium sulfate (DSS). Compared with control mouse colons, KRS-positive cancer regions in colons from AOM-DSStreated animals showed GAS6 expression and recruitment of CD206-positive M2 macrophages, but not CD11c-positive M1 macrophages (Figure 9A). Using $\mathrm{Krs}^{-/+}$knockout mice, recruitment of CD206-positive M2 macrophages to KRS-positive cancer regions was further found to be KRS-dependent (Supplemental Figure 7). To evaluate in vivo metastatic potential, mice were subjected to tail vein injection of KRS-positive shControl or KRSsuppressed (shKRS\#2) CT26 cells (Supplemental Figure 8A). Lung tumor formation was found to be obviously dependent on KRS expression (Supplemental Figure 8, B and C). The expression of GAS6 and the neighboring CD206-positive M2 macrophages, but not CD11c-positive M1 macrophages, populations closely correlated with KRS-positive tumor regions (Supplemental Figure 8D).

To further demonstrate the metastatic potential of KRSpositive colon cancer, a colon orthotopic transplantation approach (27) using CT26 cell lines with or without KRS suppression stably transfected with a luciferase vector was adapted. Bioluminescent CT26 cells were injected s.c. in mice for 6 weeks, luminescence was measured, and syngeneic tumor cells were purified for transplantation. Colon orthotopic transplantation $(n=4)$ of CT26-shControl resulted in clear bioluminescence in the lung outside of the intestine. However, bioluminescence in the lung was not observed with transplantation of CT26-shKRS\#2 and CT26-shKRS\#5 cells (each $n=4), 3$ weeks after transplantation (Figure 9B). The luciferase signals among the stable cell lines were comparable (Figure 9B). The luciferase signals for tumor volumes before the laparotomy depended on KRS expression, and decreased after KRS or MerTK inhibition (Figure 9B). Furthermore, KRS-dependent bioluminescent metastatic tumor signals were inhibited by i.p. injection of a specific KRS inhibitor, YH16899, or a MerTK inhibitor, RXDX-106 (each $n=4$ ) (Figure 9B).

Tissue extracts from human colon cancer patients showed higher expression levels of KRS, GAS6, and laminins as well as elevated FGFR1/2 phosphorylation (Figure 9C). Further, combined analysis of gene expression from a public database (KRS and GAS6 from GEO GSE28814 data set [NCBI's Gene Expression Omnibus database]) showed that $K R S^{\mathrm{hi}} G A S 6^{\mathrm{hi}}$ colon cancer patients exhibited reduced, but statistically insignificant, metastasis-free survival $(P=0.142)$ and a significantly poor relapse-free survival $(P=0.0458)$ compared with $K R S^{\mathrm{lo}} G A S 6^{\mathrm{lo}}$ patients (Figure 10A). Additionally, concomitant $K R S^{\mathrm{hi}} F G F R I^{\mathrm{hi}}$ colon cancer patients showed significantly reduced metastasisfree survival $(P=0.00756)$ and poor relapse-free survival $(P=$ 0.00265) (Figure 10A). Across patients with varying stages of colon cancer, KRS immunostaining overlapped with immunostaining for CD206 and GAS6 in cancer tissues (Figure 10B). Altogether, these results suggest that M2 macrophages rather than M1 macrophages are proximal to KRS-positive colon cancer masses, and potentially have a stimulatory influence on invasive cancer migration and successful tumor metastasis.

Positive crosstalk between KRS-positive colon cancer cells and M2 macrophages remodelsthemicroenvironment for KRS-dependent metastatic phenotypes. Communication between KRS-positive cancer cells and neighboring macrophages can influence the microenvironment in a way that supports KRS-mediated metastatic phe- 
$\mathbf{A}$

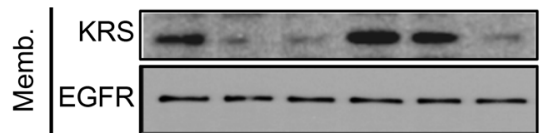

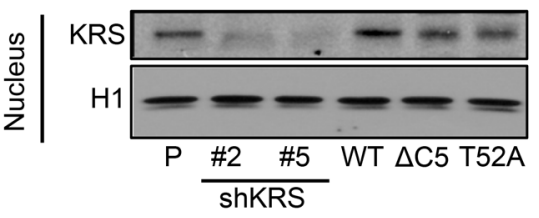

HCT116

C
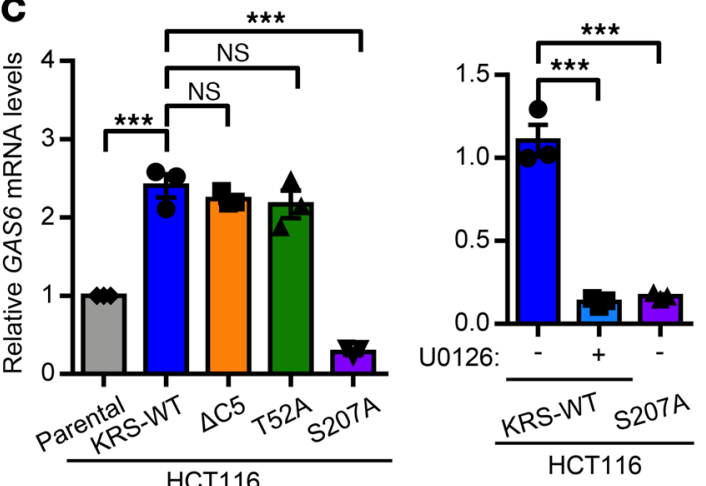

B

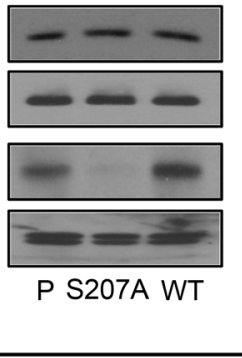

B

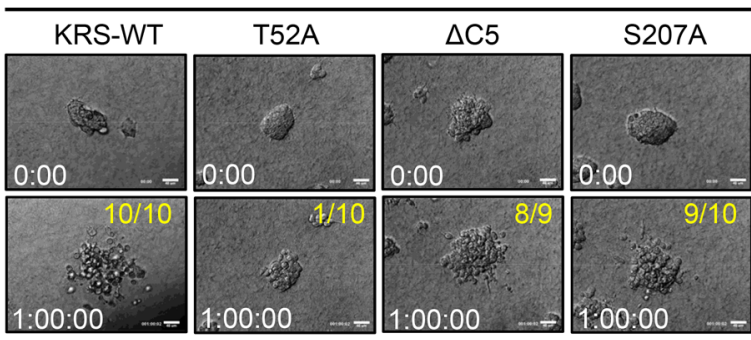

D

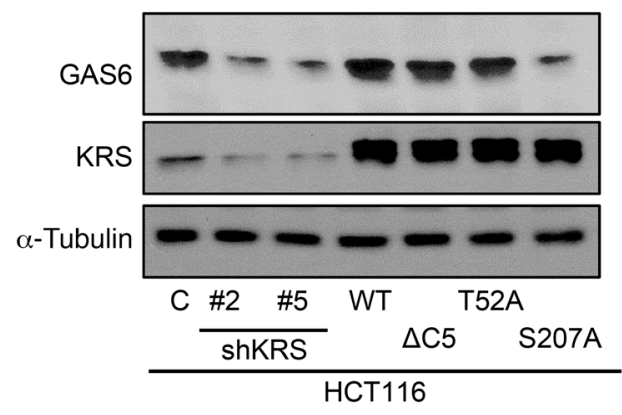

E

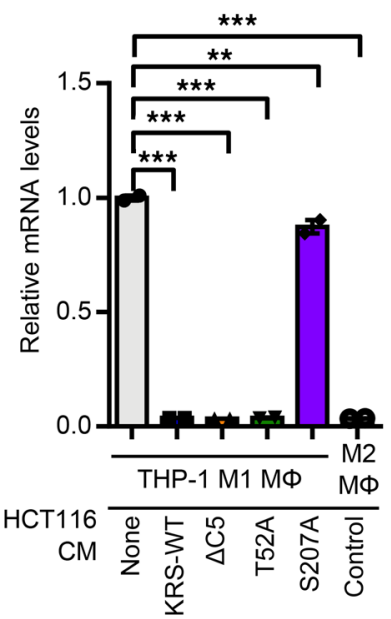

ITGAX (CD11C)

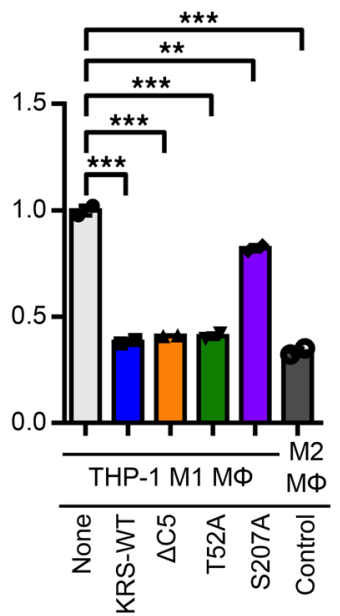

IL1A

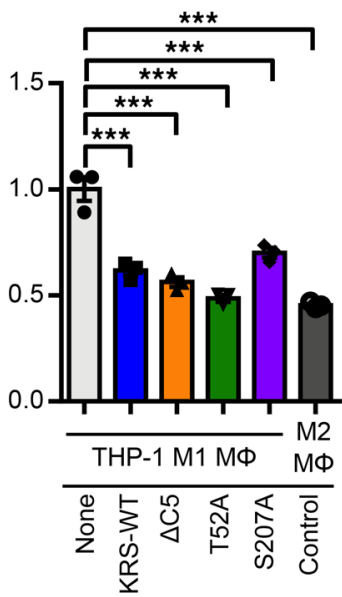

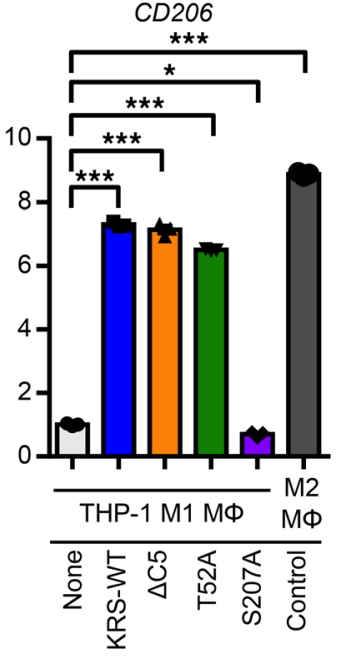

$\mathbf{F}$

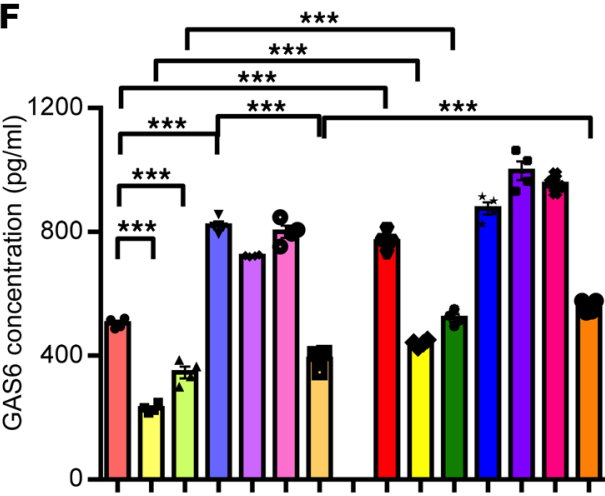

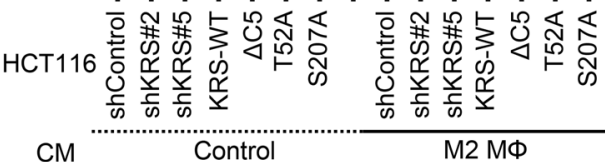

Figure 6. Nuclear KRS causes M2 macrophage polarization. (A) Plasma membrane and nucleus fractions were prepared from HCT116 spheroids, before immunoblotting. (B) HCT116 spheroids expressing exogenous KRS-WT or KRS point mutants were time-lapse-imaged for 1 day $\left(1^{\mathrm{d}}: 00^{\mathrm{h}}: 00^{\mathrm{min}}\right)$. Scale bars: $40 \mu \mathrm{m}$. (C) The GAS6 mRNA levels from the 3D HCT116 spheroids in the absence or presence of a specific MEK/ERK inhibitor (U0126) or with the KRS S207 mutant (right) were measured by real-time PCR. (D) HCT116 spheroids in 3D gels were processed for immunoblots. (E) The CM from HCT116 spheroids were administered to $\mathrm{M} 1$ or $\mathrm{M} 2$ macrophages before determination of the TNFA, ITCAX (CD11C), IL1A, and CD206 mRNA levels. (F) The 3D HCT116 spheroids were treated with THP-1 CM (Control) or THP-1 M2 macrophages for 24 hours before GAS6 ELISA analysis. The data are presented as the mean $\pm \mathrm{SD} .{ }^{*} P<0.05,{ }^{* *} P<0.01,{ }^{* * *} P<0.001$ by 1 -way ANOVA with Dunnett tests $(\mathbf{C}, \mathbf{E}$, and $\mathbf{F})$. The data shown represent 3 different observations. 
A

Region 3

Region $1 \quad(-1473 /-1271 b p)$ Region 2
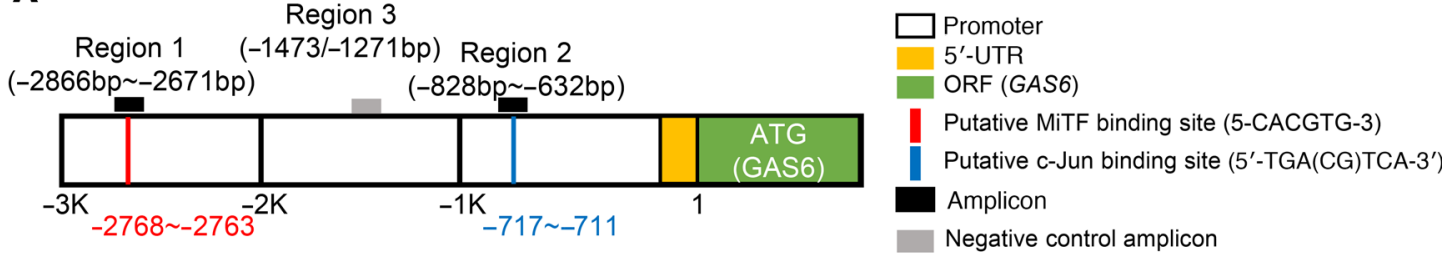

\section{B}

R1

R3
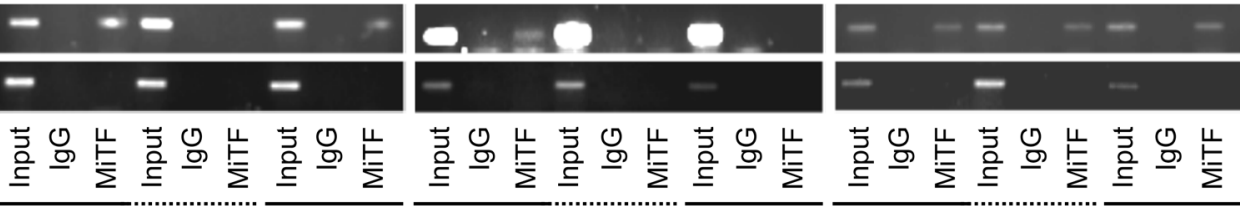

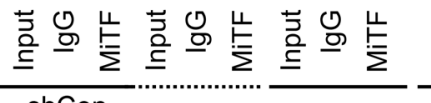

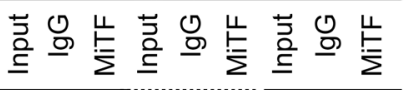

shCon ${ }_{S h K R S \# 2} \frac{{ }_{S h K R}}{M 2 M \Phi C M}$

shCon ${ }_{S h K R R^{2}}{ }_{S h K R S R^{5}}$

$\frac{{ }_{S h K R}{ }_{S h}{ }^{S h} K R^{S \# 5}}{M 2 M \Phi C M}$
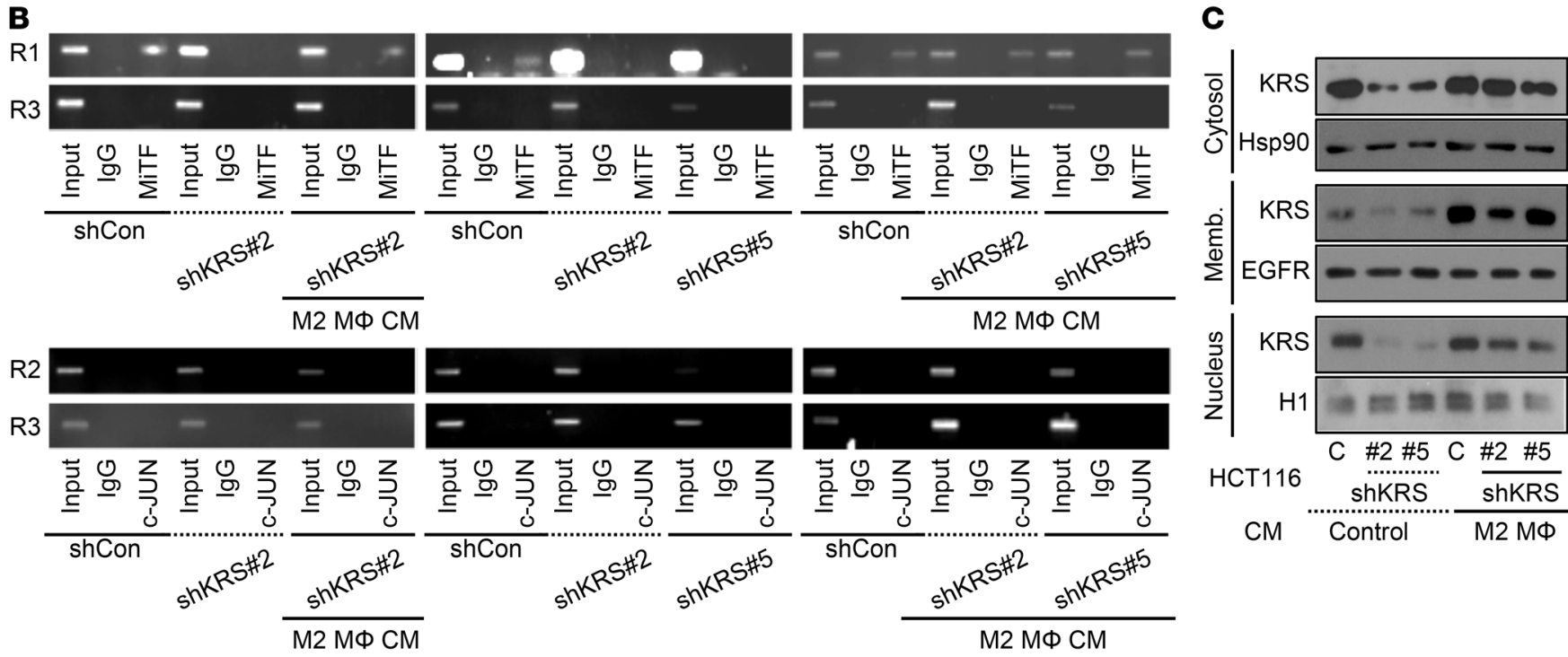

HCT116

CM

Control $\frac{\overline{\text { shKRS }}}{\text { shKRS M }}$

D

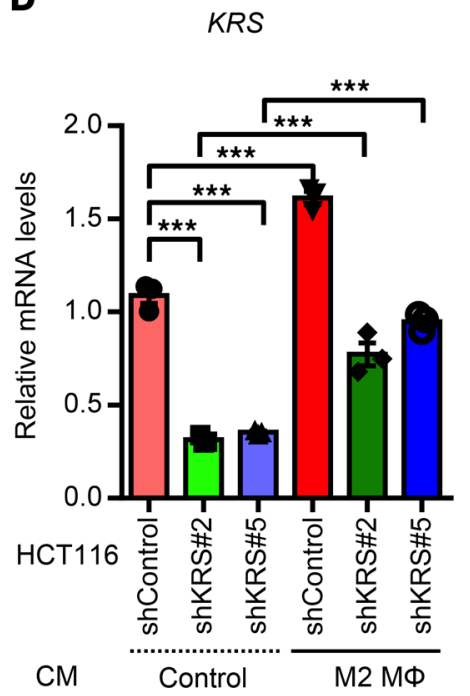

KRS

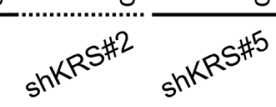

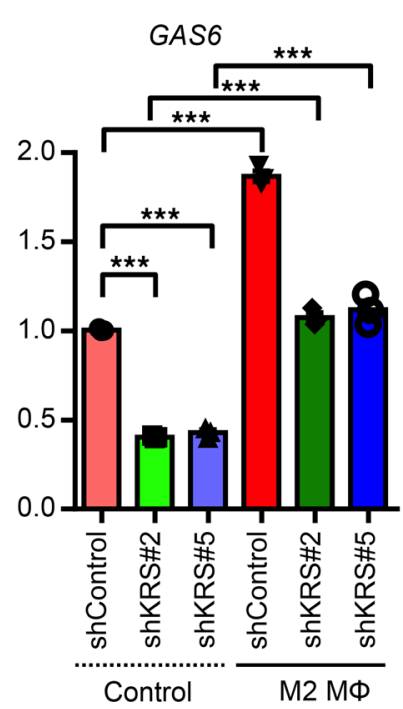

E

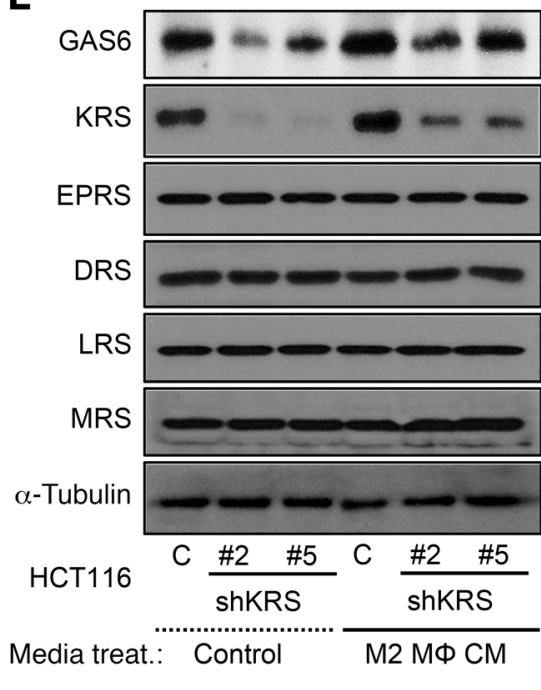

Figure 7. Nuclear KRS causes transcriptional activation of the GAS6 promoter regions by MiTF. (A) Putative MiTF or c-Jun binding sites in the GAS6 promoter region. (B) HCT116 spheroids embedded in 3D collagen I gels for 24 hours (shControl, shKRS\#2, and shKRS\#2 treated with M2 macrophage-CM) were processed for ChIP analysis. Antibodies against MiTF or C-Jun were used to examine binding to regions (1, 2, and 3 ) in the GAS6 promoter. (C-E) HCT116 spheroids in 3D collagen I gels were treated with the CM of control THP-1 or M2 macrophages, before analysis of KRS expression following cellular fractionations (C), mRNA levels of KRS or GAS6 (D), and protein levels of GAS6 and other aminoacyl-tRNA synthetases (E). EPRS, glutamyl-prolyl-tRNA synthetase; DRS, aspartyl-tRNA synthetase; LRS, leucyl-tRNA synthetase; MRS, methionyl-tRNA synthetase. The data are presented as the mean \pm SD. ${ }^{* * *} P<0.001$ by 1 -way ANOVA with Dunnett tests (D)., The data shown represent 3 different observations. See also Supplemental Figure 6.

notypes. Membranous KRS requires laminin for the laminin receptor (p67LR), which binds KRS and integrin to activate intracellular ERK signaling (12). Laminin and other components of the ECM can be secreted by CAFs (28). Therefore, we examined whether the communication between KRS and microenvironmental components could lead to ECM remodeling. To do so, we investigated whether normal colon fibroblasts (CCD-18Co) could be activated to induce ECM remodeling as a result of communication between KRS-positive colon cancer spheroids and M2 macrophages. CM from THP-1 M2 macrophages but not from M1 macrophages or monocytes induced CAFs to express laminin and caused morphological changes in the cells (polygonal 
A

Macrophage
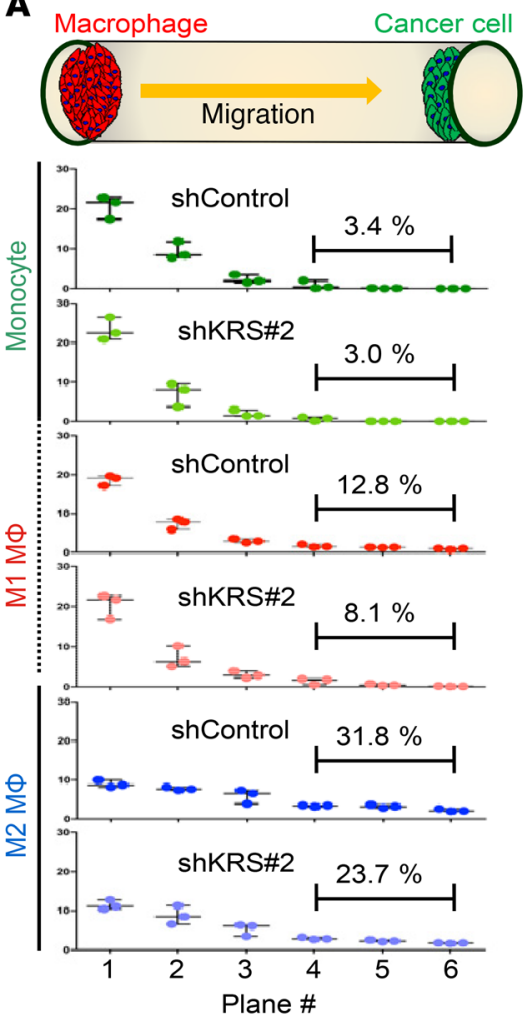

C
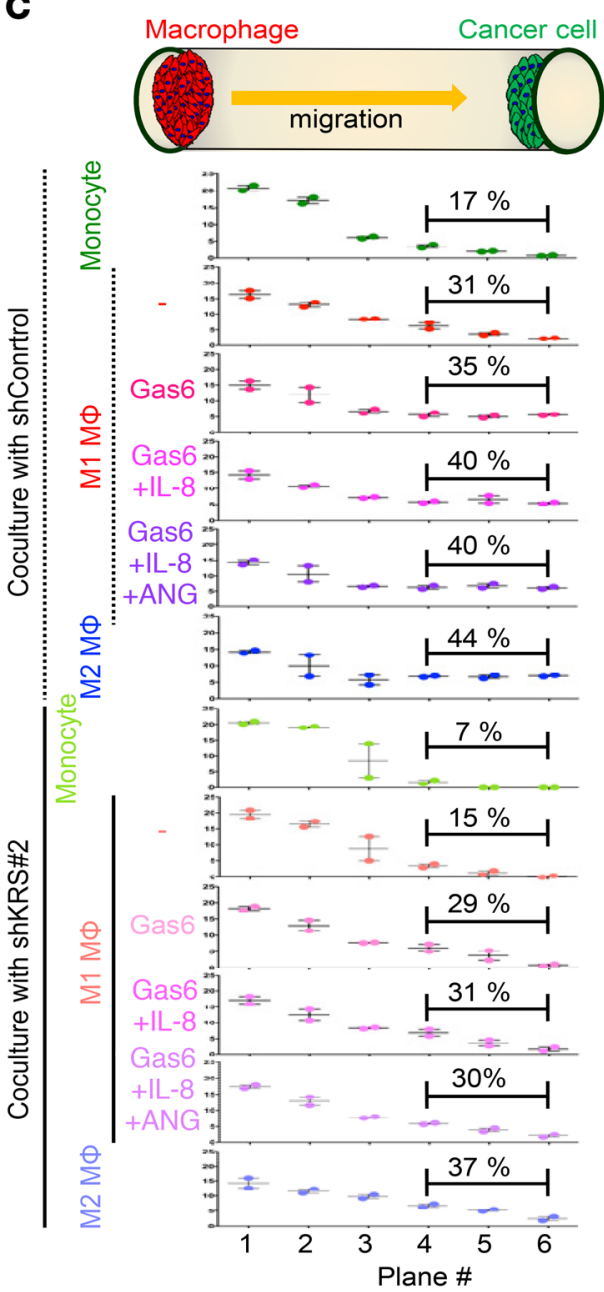

B

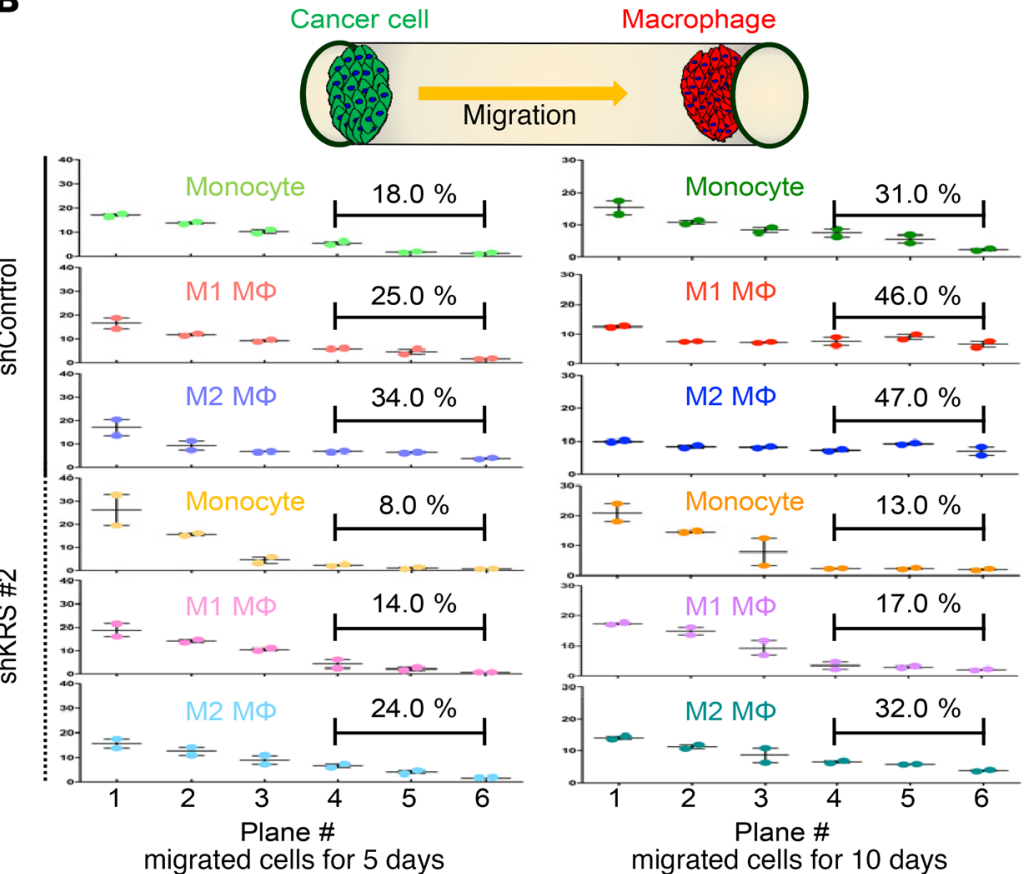

migrated cells for 5 days

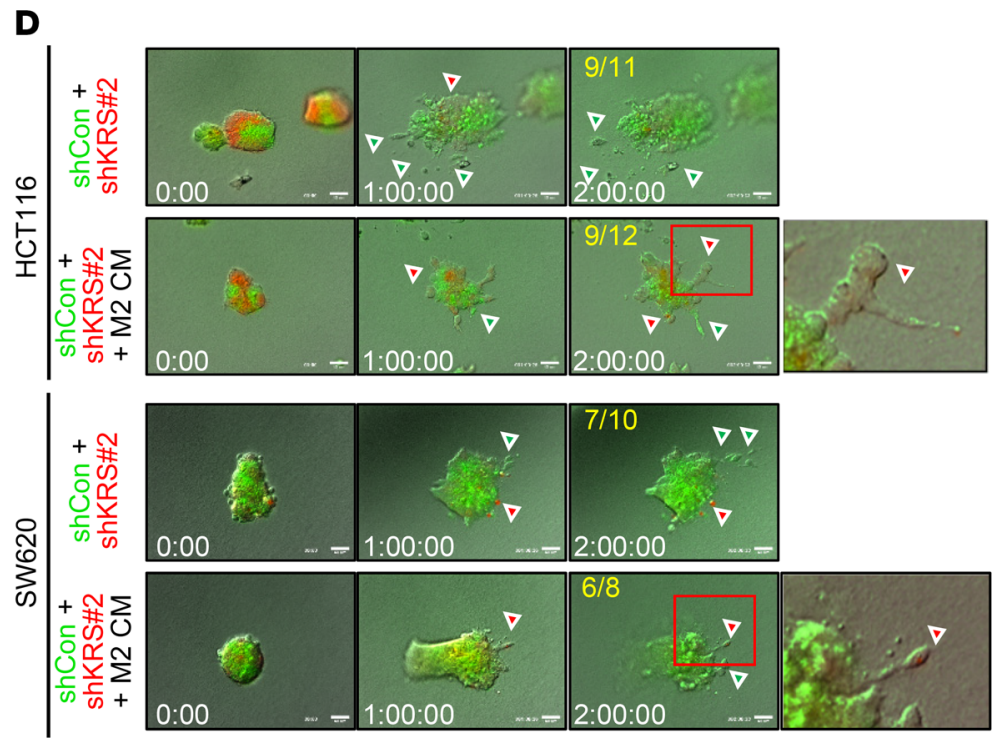

Figure 8. KRS-positive spheroids and tumor masses prefer to crosstalk with M2 macrophages. (A-C) A high-content screening (HCS) platform was processed for migration of M2 macrophages toward KRS-positive (shControl) cells for 7 days (A), migration of KRS-positive cells toward M2 macrophages for 5 or 10 days (B), and migration of M2 macrophages or M1 macrophages that were treated with nothing (-), GAS6, GAS6 + IL8, or GAS6 + IL-8 + ANG toward KRS-positive (shControl) cells and toward KRS-suppressed (shKRS\#2) cells for 10 days (C). (D) Coculture of KRS-positive (green) and KRS-suppressed (red) colon cancer cells in spheroids was done before time-lapse imaging for 2 days to observe invasive outgrowth upon treatment with CM from M2 macrophages. Data shown represent 3 independent experiments. Scale bars: $40 \mu \mathrm{m}$. 
A

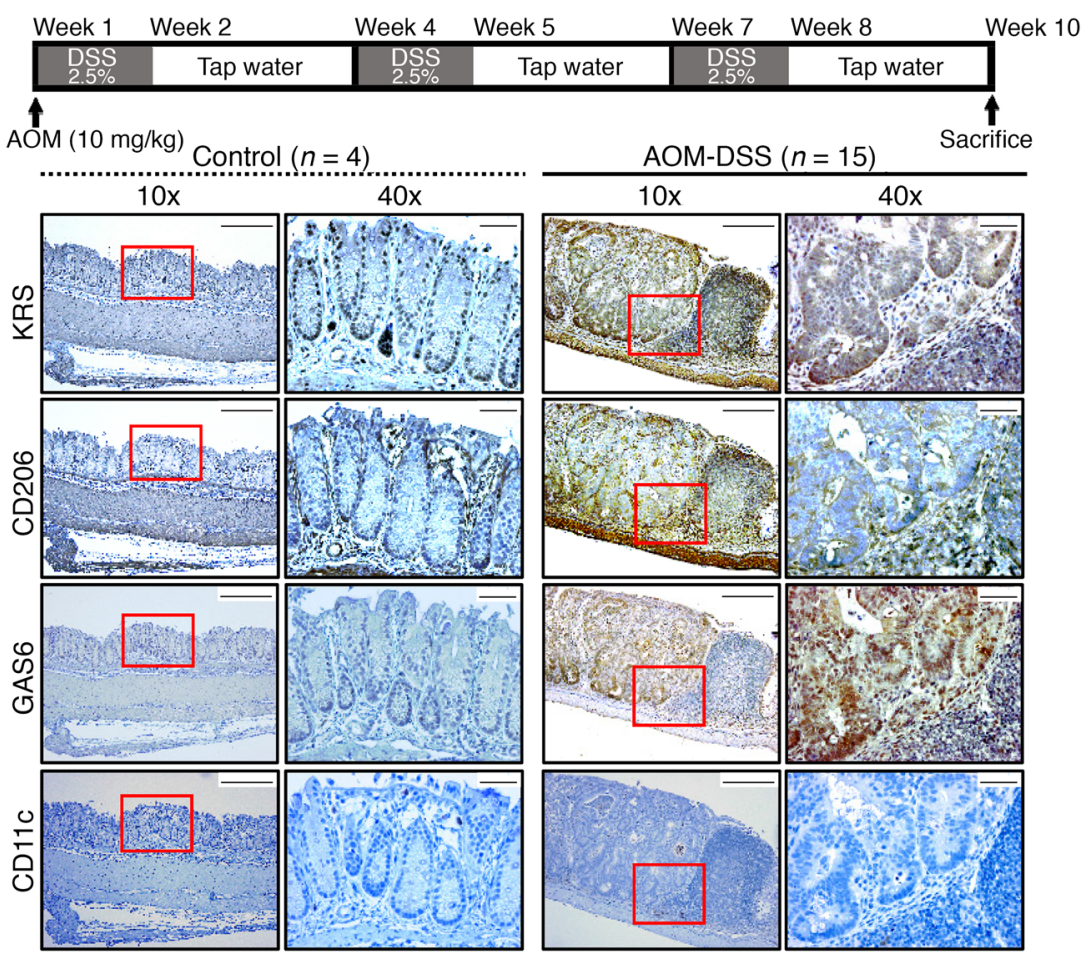

B

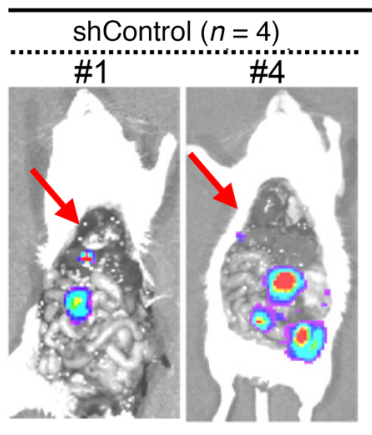

Vehicle $(n=4)$
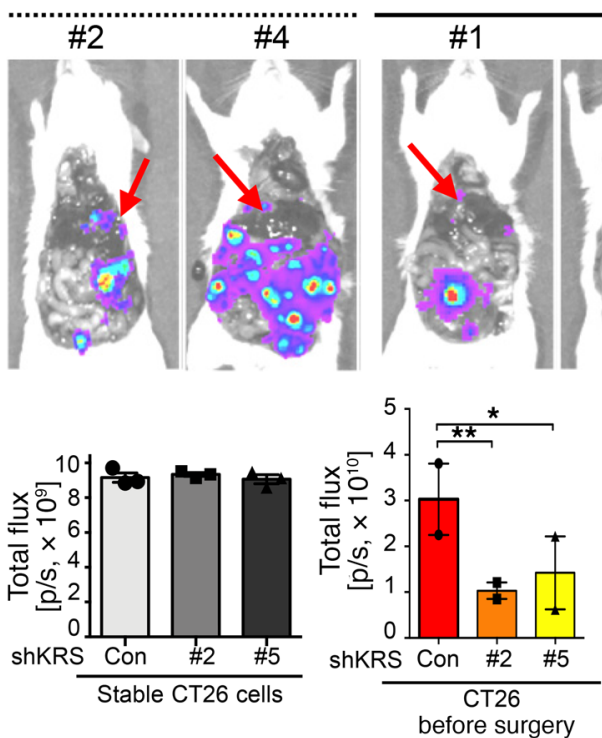

CT26

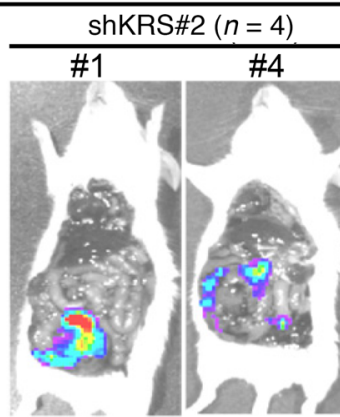

YH16899 $(n=4)$

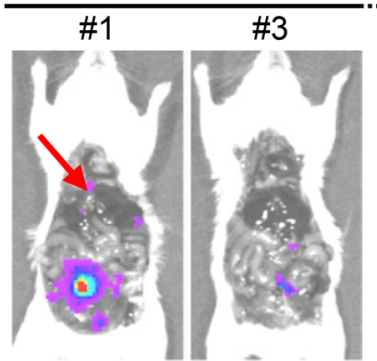

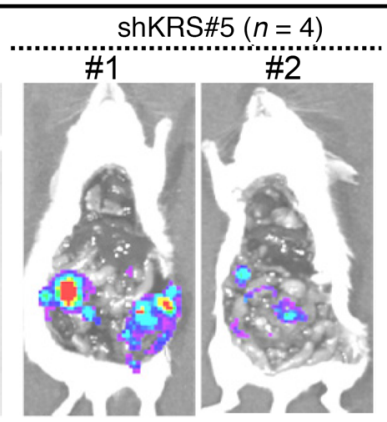

YH16899 $+\operatorname{RXDX}-106(n=4)$

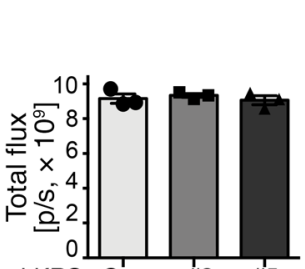

shKRS Con \#2 \#5
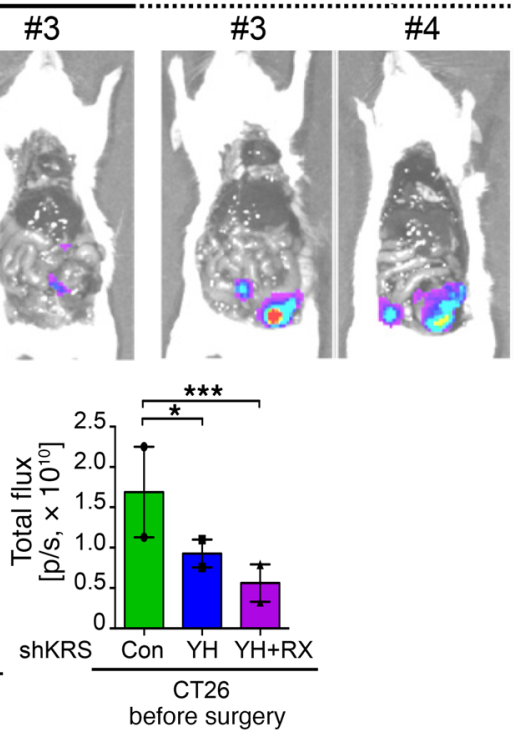

C

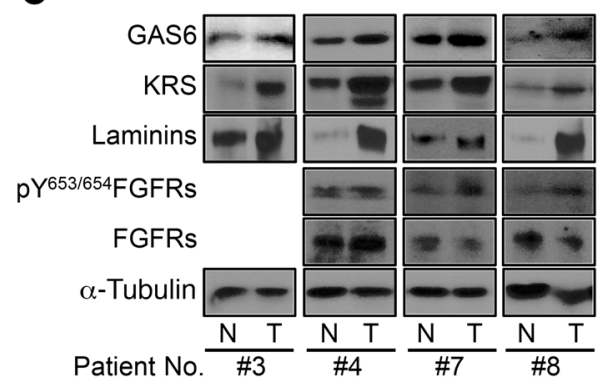

Figure 9. Significance of KRS in colon cancer metastasis. (A) Colon tissues from control $(n=4)$ and AOM-DSSinduced cancer models $(n=15)$ of 7-week-old BALB/c male mice were processed for immunostaining. Scale bars: $100 \mu \mathrm{m}$. (B) The CT26 cells stably infected with pMSCV-Luc2 retrovirus were injected s.c. into 8-week-old BALB/ c-nude female mice. Luciferase-positive cells (top left graph) were subsequently purified from the syngeneic tumors. The cells were orthotopically transplanted to the colon ( $n=4$, top) of 6 -week-old $\mathrm{BALB} / \mathrm{c}$ female mice. One week later, vehicle or specific inhibitors ( $n=4$, bottom) were administered i.p. every other day, and 3 weeks later the luciferase signals were analyzed before (right graphs) or after (representative animal images) laparotomy. The data are presented as the mean $\pm \mathrm{SD}$. ${ }^{*} P<0.05$, ${ }^{* *} P<0.01$ ${ }^{* *} P<0.001$ by 1 -way ANOVA with Dunnett tests. (C) Normal or clinical colon cancer patient tissues were immunoblotted for the indicated molecules. See also Supplemental Figures 7 and 8.

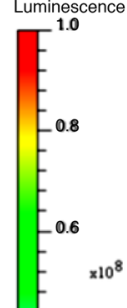

$-0.4$ $-02$

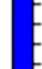

Radiance (p/sec/cm $/ \mathrm{sr}$ ) Color scale
Min-1.50e6 Max-1.00e8 
A

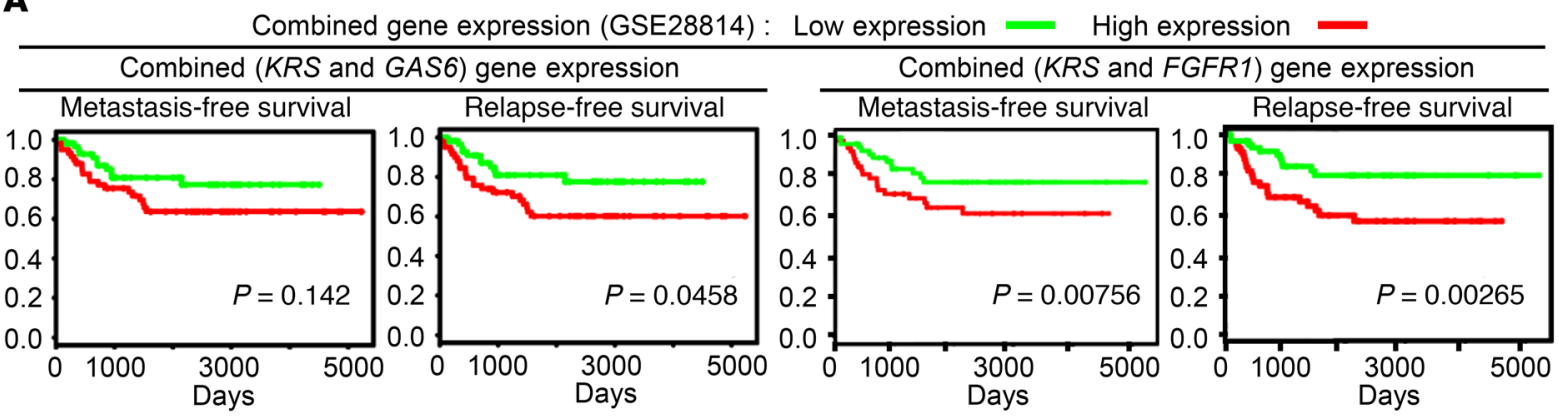

B

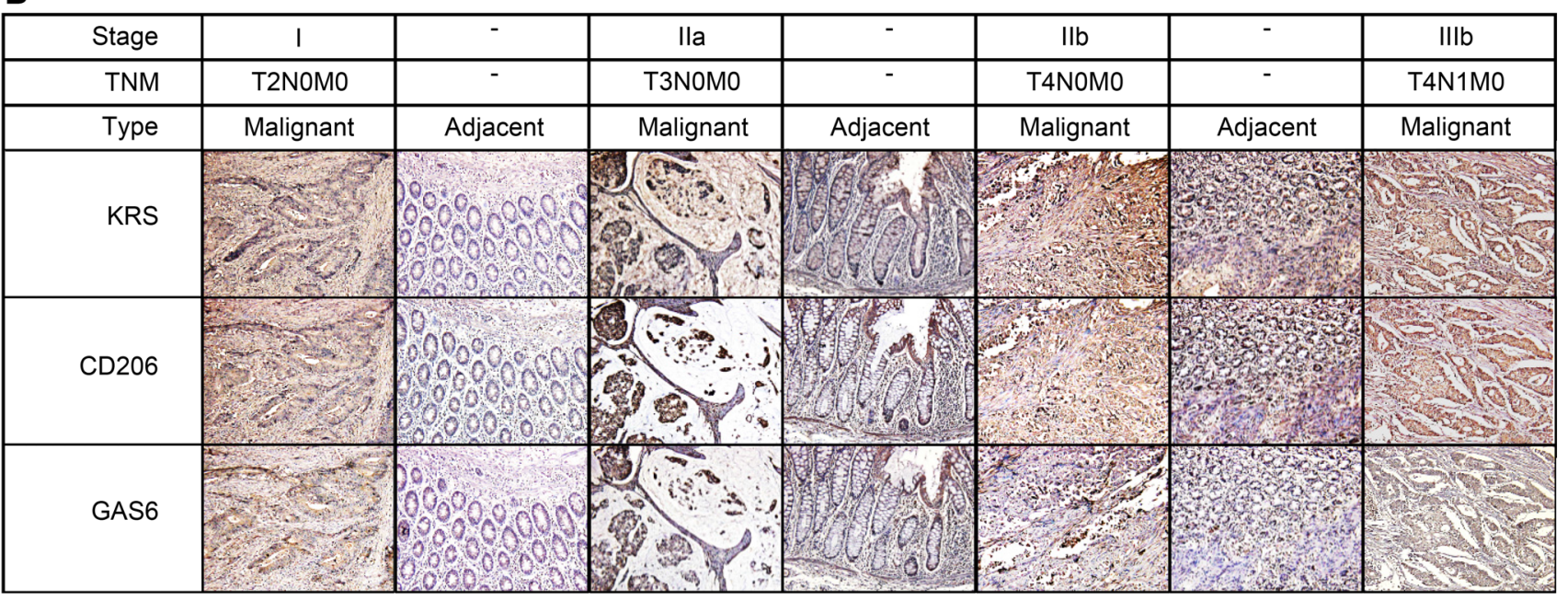

Figure 10. Clinical significance of KRS in colon cancer progression and metastasis. (A) Combined gene expression analysis of KRS and either GAS6 or FGFR1 from public data (GSE28814) for metastasis-free survival or relapse-free survival. (B) Clinical colon cancer patient tissues were immunostained for the indicated molecules. fData shown represent 3 independent experiments. Original magnification, $\times 40$.

shapes) (Figure 11A). However, CM from M1 macrophages that were pretreated with $\mathrm{CM}$ from KRS-positive colon spheroids also caused CAFs to express laminin and induced morphological changes. This effect was lost in response to CM from M1 macrophages that were pretreated with CM from KRS-suppressed spheroids (Figure 11B). To determine whether the effects on CAFs were at the transcriptional level, mRNA levels of laminin $\alpha 1$ (LAMA1), laminin $\beta 1$ (LAMB1), and fibronectin (FN1) were evaluated. CM from M2 macrophages but not from the controls, monocytes, or M1 macrophages dramatically increased LAMA1, $L A M B 1$, and FN1 mRNA levels (Figure 11C, first 4 bars), suggesting an environment enriched with the p67LR ligand: laminin consisting of laminin $\alpha 1$ and $\beta 1$. These mRNA levels were also elevated after treatment with CM from M1 macrophages when CM were prepared from M1 macrophages pretreated with KRSpositive colon spheroids (shControl or KRS-WT) but not KRSsuppressed (shKRS\#2) spheroids (Figure 11C). Activation of CAFs was also revealed when collagen I contractions were examined. CM from M2 macrophages and CM from M1 macrophages that had been pretreated with KRS-positive (shControl or KRS-WT) spheroids caused contractions of the collagen I gels, but CM from monocytes and M1 macrophages did not (Figure 11D). CM of M1 macrophages that had been treated with GAS6, IL-8, and ANG resulted in activation of CAFs similar to that caused by M2 macrophage-CM (Figure 11E).
Dissemination was analyzed in KRS-positive HCT116 cells (labeled with green fluorescent dye) cocultured with CCD-18Co fibroblasts (labeled with red fluorescent dye). Inactive CAFs without any CM pretreatment showed basal levels of KRSdependent outgrowth of shControl KRS-positive spheroids. In contrast, coculturing with CAFs pretreated with $\mathrm{CM}$ from M2 macrophages caused dramatic dissemination of shControl spheroids (Figure 11F). Thus, the effects of M2 macrophagemediated CAF activation on environmental remodeling via laminin synthesis were more important for KRS-dependent migration of cells, presumably because membranous KRS binds to p67LR in a laminin environment (6).

\section{Discussion}

KRS is an enzyme that participates in protein translation (29). Beyond its translational role, evidence in this study suggests that KRS supports communication between cancer cells and neighboring cells, including macrophages and CAFs, during invasive outgrowth of metastatic cells from colon cancer spheroids. The communication attributed to the close proximity of the cell types and to the expression of soluble cytokines or factors is dependent on KRS expression in cancer spheroids. KRS-positive spheroids, embedded in 3D collagen I gels, secreted cytokines, including GAS6, IL-8, and ANG, that caused M2 macrophages infiltration and/ or polarization. Nuclear KRS was critical for the MiTF-mediated 
transcription and expression of GAS6, which then caused the M2 polarization of macrophages. Pharmacological inhibition of KRS or MerTK in M2 macrophages led to reduced metastasis of KRSpositive cells that were transplanted orthotopically in the colon of mice. M2 macrophages were located in close proximity to cancer masses and secreted soluble factors, including FGF2, GRO $\alpha$, and M-CSF. These factors activated intracellular signaling molecules (FGFR1/2, ERKs, and STAT3) within the cancer spheroids and caused MMP9 expression, paxillin activation, and metastatic cell migration. M2 macrophages restored KRS expression and disseminative phenotypes in KRS-positive spheroids and, to a lesser extent, even in KRS-suppressed spheroids. Therefore, colon cancer spheroids and neighboring M2 macrophages formed a KRSdependent positive-feedback loop. In addition, CAFs were involved in the communication between KRS-positive cancer spheroids and M2 macrophages. The secretion of laminins by CAFs was activated by communication between cancer spheroids and macrophages, and resulted in more dramatic disseminative phenotypes, likely because the membranous KRS of the spheroids is favored by lami$\operatorname{nin}(6,12)$. These results suggest that KRS plays an important role in remodeling the tumor microenvironment during invasive cell outgrowth from colon cancer spheroids (Figure 12).

Interestingly, we found that invasive outgrowth from spheroids required KRS expression both on the plasma membrane and in the nucleus. Membranous KRS binds to the laminin receptor (p67LR) together with $\alpha_{6} \beta_{1}$ integrin, depending on the availability of laminins in the extracellular space $(6,12)$. Although this study used pan-laminin antibodies for immunoblots and immunostainings, colon or colorectal cancer is known to aberrantly express laminin $\alpha 1, \alpha 5, \beta 1$, and $\beta 3$ chains (30). Meanwhile, CAFs that were activated by $\mathrm{M} 2$ macrophages caused induction of FN1, $L A M A 1$, and $L A M B 1$ mRNAs in this study, presumably resulting in a microenvironment enriched with their protein products. Therefore, the membranous KRS complex is stabilized at the plasma membrane and triggers intracellular signaling, which can activate cell-ECM interaction during invasive migration (8). Unlike the spheroids expressing endogenous or exogenous KRS, we showed that spheroids stably expressing the KRS T52A mutant, which is unable to move to the plasma membrane, did not cause invasive migration. Furthermore, small compounds that interfere with the formation of membranous KRS-containing complexes abolish KRS-mediated colon cancer metastasis in animal models (9). Similarly, dissemination of endogenous KRS-positive spheroids with or without treatment with CM from M2 macrophages was blocked by concomitant treatment with an anti-membranous KRS $\left(\alpha-\mathrm{KRS}_{\text {mem }}\right)$ antibody, presumably because this treatment disturbed the KRS-mediated protein complex formation on the cell surface and thereby disrupted intracellular signal transduction.

The membranous KRS-containing protein complex could also include other receptors, such as FGFR1/2 and $\alpha_{v} \beta_{3}$ integrin receptor. This is probable because FGF2 secreted by M2 macrophages activated the FGFR1/2 of cancer spheroids in a KRS-dependent manner (Figure 2E), and FGF2 and FGFR1/2 are shown to bind to $\alpha_{\mathrm{v}} \beta_{3}$ integrin at focal adhesions (20). The KRS-containing protein complex responsive to FGF2 that was secreted by the M2 macrophages could be stabilized on the plasma membrane of cancer cells in a laminin-containing environment. This probable event could transduce the intracellular signaling activity of ERKs, paxillin (12), STAT3 (this study), and NF- $\kappa$ B (unpublished observation). Alternatively, in KRS-suppressed cells, $\alpha_{\mathrm{v}} \beta_{3}$ integrin association with FGF2/FGFRs may transduce intracellular signaling to ERKs, STAT3, and others. Thus, neutralization of $\alpha_{v} \beta_{3}$ integrin or membranous KRS could result in partial inhibition of the invasive outgrowth of 3D HCT116 spheroids that involves KRS-dependent or FGF2/FGFR2-dependent regulatory mechanisms. In the case of KRS-suppressed cells, M2 macrophage-CM treatment could promote KRS expression and allow association of FGF2/FGFRs with $\alpha_{v} \beta_{3}$ integrin for invasive migration from cancer spheroids. The membranous KRS-dependent signaling activities could then lead to morphological adaptations and changes in the adhesion properties necessary for invasive outgrowth from cancer spheroids.

KRS can dynamically translocate to other cellular spaces, such as the extracellular space and the nucleus. When KRS-positive or KRS-suppressed HCT116 spheroids were treated with recombinant KRS (a secreted form; ref. 31), the spheroids did not respond by enhancing dissemination (data not shown). However, the recombinant KRS protein level used may have been too low to elicit an effect. The spheroids expressing the $\Delta \mathrm{C} 5$ mutant KRS protein, which is unable to be secreted, still exhibited dissemination, suggesting that KRS secretion may not be critical for the disseminative phenotypes. However, spheroids expressing the S207A mutant KRS protein, which is unable to traffic into the nucleus, still showed invasive migration. This is presumably because membranous KRS was still available to function basally during the dissemination process. However, the S207A mutant spheroids could not induce GAS6 transcription and expression, leading to a lack of M2 macrophages polarization (Figures 6 and 7). This lack of polarization prevented remodeling of the microenvironment, and resulted in less efficient metastatic migration.

In this study, MiTF was recruited to the promoter region of GAS6 in colon spheroids in a KRS-dependent manner. Similarly, the phosphorylation of KRS at S207 by ERKs in mast cells allows KRS trafficking to the nucleus and MiTF activation for Ap4A transcription (26). Interestingly, M2 macrophage-CM treatment of KRS-suppressed spheroids caused recruitment of MiTF to the GAS6 promoter regions for GAS6 transcription. This process concomitantly increased KRS expression in spheroids. TNF- $\alpha$, a marker of M1 macrophages, causes the secretion of KRS from colon cancer cells without the de novo synthesis of KRS (31). In our study, CM from M2 macrophages contained factors that activated $K R S$ transcription. However, it remains to be determined how CM from M2 macrophages upregulates KRS levels. After S207 phosphorylation of KRS by ERKs and entry into the nucleus, KRS activated MiTF to induce transcription of GAS6, eventually facilitating the M2 polarization of neighboring macrophages. Our study thus provides evidence for a noncanonical role of nuclear KRS in transcriptional processes during the remodeling of the tumor microenvironment. In addition to its role in transcription, the initiation of intracellular signaling activity by membranous KRS is important for its function in facilitating cell migration.

Among the cytokines induced by KRS-positive spheroids in our study, GAS6 was more important than IL-8 or ANG for the polarization of M1 to M2 macrophages and the infiltration of M2 macrophages toward cancer spheroids. GAS6 is a ligand for the 


\section{A}
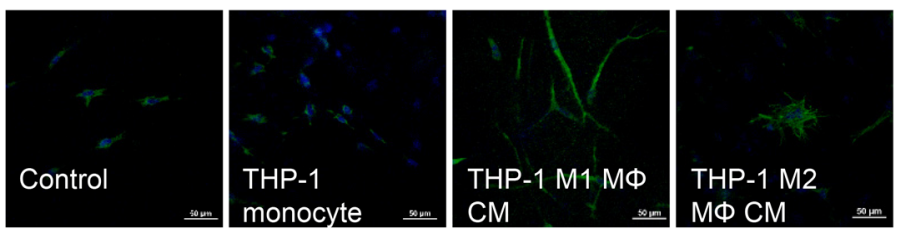

B

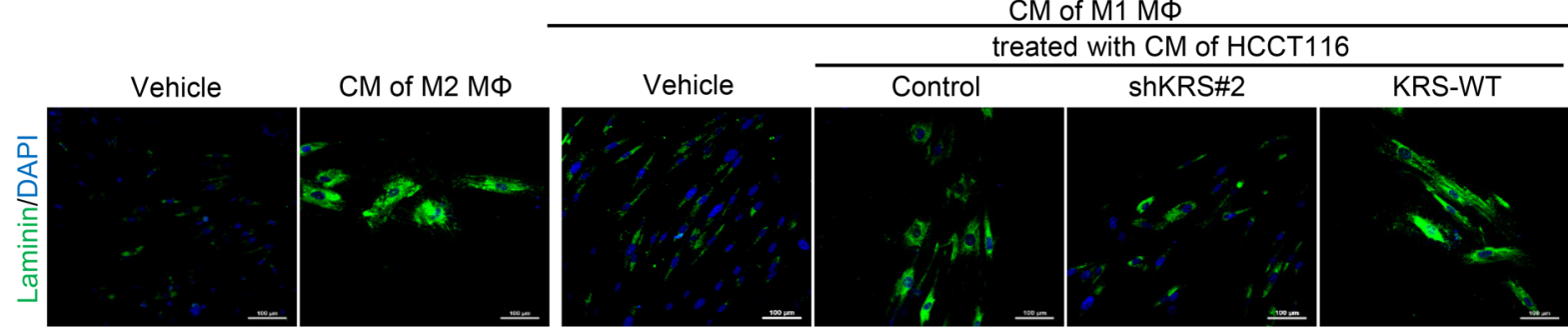

C

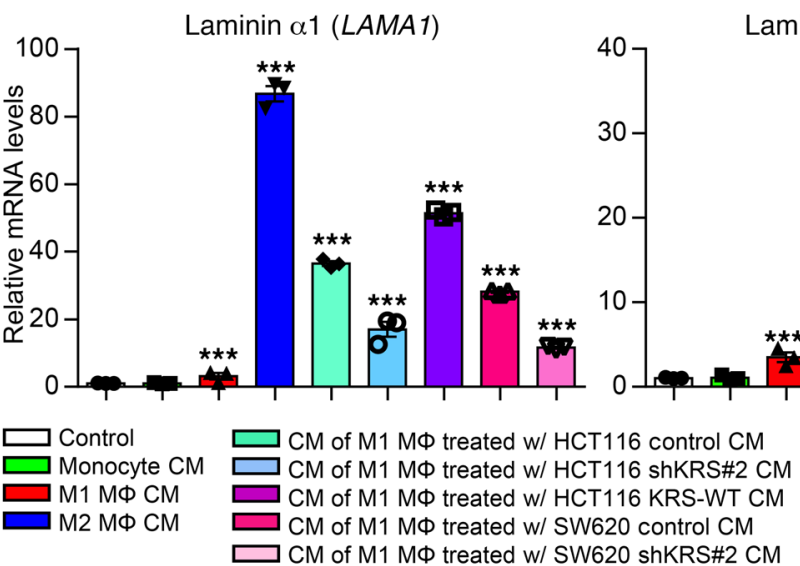

D

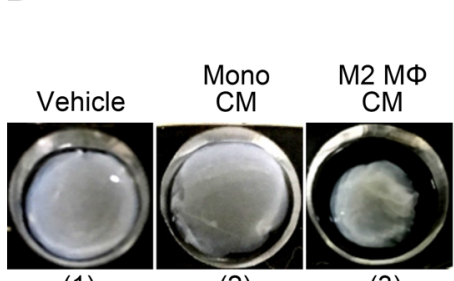

(1)
(2)

(3)

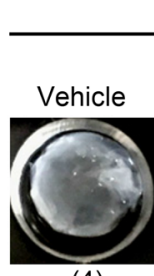

(4)

M1 MФ CM

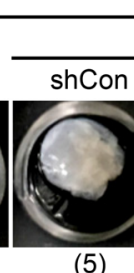

(5)

(6)

E

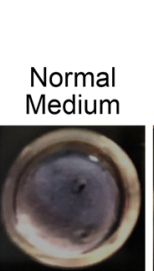

(1)

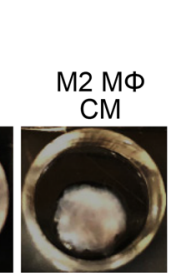

(2)

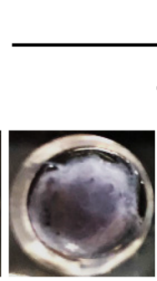

(3)

M1 МФ CM

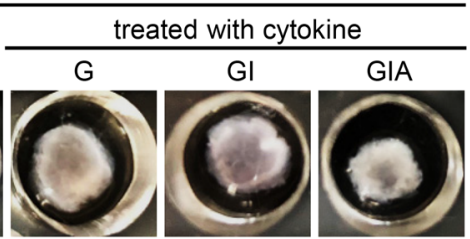

(5)
(6)

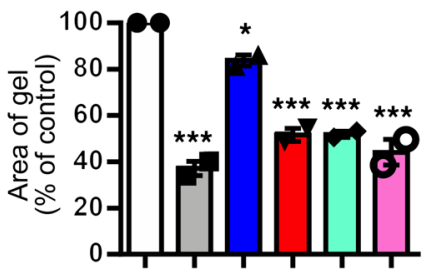

(1) (2) (3) (4) (5) (6)

$\mathbf{F}$

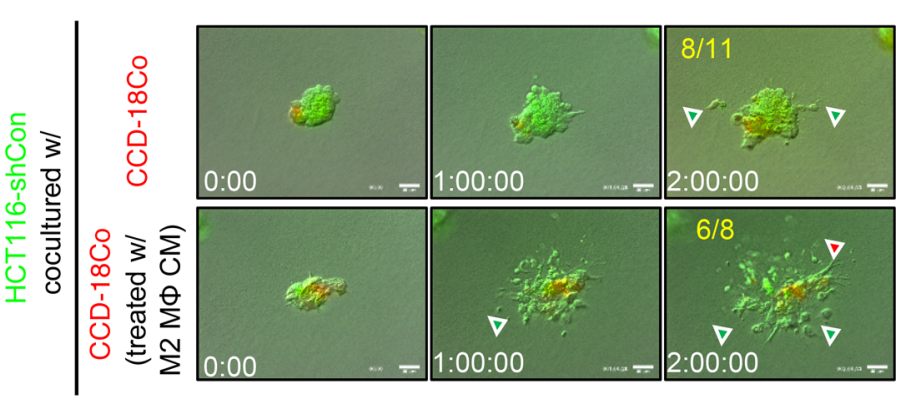

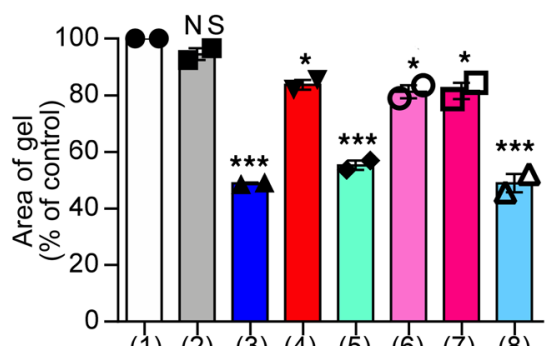

$\begin{array}{llllll}\text { (1) } & \text { (2) (3) (4) (5) } & \text { (6) } & \text { (7) } & \text { (8) }\end{array}$ 
Figure 11. A positive relationship between KRS-positive colon cancer cells and an M2 macrophage-remodeled microenvironment promotes KRS-dependent metastasis. (A) Normal human colon fibroblast (CCD-18Co) cells in 3D collagen I gels were analyzed for laminin expression (green) following treatment with CM of THP-1 monocytes or M1 or M2 macrophages. Nuclei were stained with DAPI (blue). (B-D) CCD-18Co fibroblasts were treated with vehicle, M2 macrophage-CM, or M1 macrophage-CM that had been treated with vehicle or CM from HCT116 spheroids (shControl, shKRS\#2, or KRSWT) for 4 days. Immunostaining was done for laminin (green), and nuclei were stained with DAPI (blue) (B). Laminin $\alpha 1$ (LAMA1), laminin $\beta 1$ (LAMB1), or fibronectin (FN1) mRNA levels (C) or collagen contraction (D) were analyzed. (E) Collagen gel contraction was analyzed for CCD-18Co fibroblasts in normal media, CM from M2 macrophages, or CM from M1 macrophages that had been treated with GAS6 (G), GAS6 + IL-8 (GI), or GAS6 + IL-8 + ANG (GIA) for 4 days. (F) KRS-positive (shCon, shControl) colon cancer spheroids (green) were cocultured with CCD-18Co fibroblasts (red) that were treated with or without $\mathrm{M} 2$ macrophage-CM, before imaging of disseminative outgrowth of cancer cells and fibroblasts. The data are presented as the mean $\pm \mathrm{SD} .{ }^{*} P<0.05,{ }^{* *} P<0.001$ by 1 -way ANOVA with Dunnett tests (C-E). The data shown represent 3 independent experiments.

receptor MerTK that is restricted to macrophages (25). Binding of GAS6 to MerTK on M1 macrophages decreases levels of IL-12 (an M1 macrophage marker), increases response to dying cells, and suppresses macrophage inflammatory response (32). The phagocytosis of dying cells also drives M2 polarization (33). Furthermore, binding of GAS6 to MerTK and the phagocytosis of dying cells increase levels of IL-10 (an M2 macrophage marker), leading to increases in antiinflammatory and protumorigenic macrophages (34). GAS6, which was secreted by KRS-positive spheroids, also increased the phosphorylation of MerTK in M1 macrophages in this study, suggesting that its activation was necessary for M2 macrophage polarization. Therefore, membranous and nuclear KRS in colon cancer cells could be critical for communication between cancer spheroids and macrophages and may promote KRS-dependent dissemination from the spheroids.

M1 macrophages are generally antitumorigenic and proinflammatory, whereas M2 macrophages are protumorigenic (35). Thus, the M2 polarization of macrophages supported by KRSpositive colon spheroids suggests a prometastatic role for KRS. This prometastatic role is supported by evidence that KRS is highly expressed in colon cancers (6). After TNF- $\alpha$ treatment, KRS is secreted to the extracellular space during inflammation $(7,31)$. KRS secretion within the lumen of exosomes from colon cancer cells triggers an inflammatory reaction (involving increased TNF- $\alpha$ secretion after 6 hours of treatment) and macrophage migration toward colon cancer cells (13). Therefore, secreted KRS may recruit M1 macrophages during early tumorigenesis. However, in this study, CM of KRS-positive cancer cells caused decreased TNF- $\alpha$ secretion from macrophages, compared with CM from KRS-suppressed cells after longer treatments for 4-10 days during macrophage differentiation. Our current study may relate to a later tumor status with a protumorigenic environment following longer periods of KRS exposure to macrophages. Thus, it is likely that the effects of KRS treatment (or expression) on macrophages can be differential depending on carcinogenesis stage. As shown in this study, the collaboration of both membranous and nuclear KRS populations caused M2 macrophage polarization and/or infiltration of M2 macrophages into KRS-positive cancer masses during later tumor stages. STAT6 is highly activated in M2 macrophages, and STAT1 is activated in M1 macrophages (21, 24). Treatment of M1 macrophages with CM from KRS-positive spheroids or with GAS6 elevated STAT6 activity, suggesting that M2 macrophage polarization occurs by KRS-dependent GAS6 induction and secretion. Increased expression of the M2 macrophage markers IL-10 and CD206 also indicated M2 macrophage polarization. Furthermore, after 10 days of incubation, M1 macrophages mimicked M2 macrophages in terms of their tendency to migrate toward KRS-positive spheroids in coculture experiments using the Operetta HCS Platform. M2 macrophages triggered the secretion of soluble factors (including FGF2, GRO $\alpha$, and M-CSF) that promoted the activation of intracellular signaling in cancer spheroids, and led to invasive outgrowth of cancer cells and activation of neighboring CAFs that then secreted laminins. The elevated laminin levels presumably provide the laminin-containing microenvironment necessary for membranous KRS-positive cancer spheroids to gain invasive migratory signaling and function.

This study provides evidence of a noncanonical role for KRS in colon cancer metastasis. Our results suggest that a positive-feedback loop between KRS-expressing cancer spheroids and diverse microenvironmental factors, including neighboring cells and soluble factors, promotes colon cancer cell dissemination. Therefore, KRSmediated intracellular signaling and the protein complexes that are critically involved in these feedback loops are promising therapeutic targets for the prevention of colon cancer metastasis.

\section{Methods}

Cells. HCT116 and SW620 human colon cancer cells and CT26 mouse colon carcinoma cells (American Type Culture Collection [ATCC]) were stably transfected with shRNA against KRS (transcript 1, NM_001130089, lysyl-tRNA synthetase MISSION shRNA Plasmid DNA; Sigma-Aldrich). Stable shControl and different stable shKRS-transfected clones were established and maintained as previously described (12). The target of shKRS\#2 is from base residue 911 to 933 of exon 7 (CCG ${ }^{911}$ GCCAGAGATACTTGGACTTGATC ${ }^{933}$ TCGAGATCAAGTCCAAGTATCTCTGGTTTTTG [exon 7]), and shKRS\#5 targets from base residue 1,071 to 1,092 of exon 12 (CCGG $^{1071}$ GCCTTTCACTTATCACAAC ${ }^{1092}$ TCGAGTTGTGATAAGTGATGAAAGGCTTTTTG [exon 12]). Lentiviral production was performed by the transfection of HEK293T cells (ATCC) using Fugene 6 (Promega) and KRS cDNA constructs in a PLKO.1 lentiviral vector backbone (6). Supernatants were collected $24-48$ hours after transfection and filtered through a $0.22-\mu \mathrm{m}$ syringe filter (Sigma-Aldrich). Colon epithelial cells were infected with lentivirus and $8 \mu \mathrm{g} / \mathrm{ml}$ Polybrene (Sigma-Aldrich) for 24 hours, and then the medium was replaced with fresh growth medium containing $1 \mu \mathrm{g} / \mathrm{ml}$ puromycin (Sigma-Aldrich) for selection. HCT116 cells overexpressing KRS were established by the stable transfection of the Myc-tagged KRS plasmid (6). The cDNA encoding a Myc-KRS S207A mutant was cloned using a QuikChange II kit (Agilent Technologies) following the manufacturer's instructions and was confirmed by direct sequencing. The HCT116 stable cell lines expressing the $\Delta \mathrm{C} 5$ and T52A KRS mutants and Myc-tagged KRS-WT that were used have been previously described $(6,31)$. Human monocyte THP-1 cells were provided by Mi-Ok Lee (Seoul National University). THP-1 cells were treated with $100 \mathrm{nM}$ phorbol-12-myristate-13-acetate (PMA) for 1 day, and the adhered cells were then differentiated into M1 mac- 


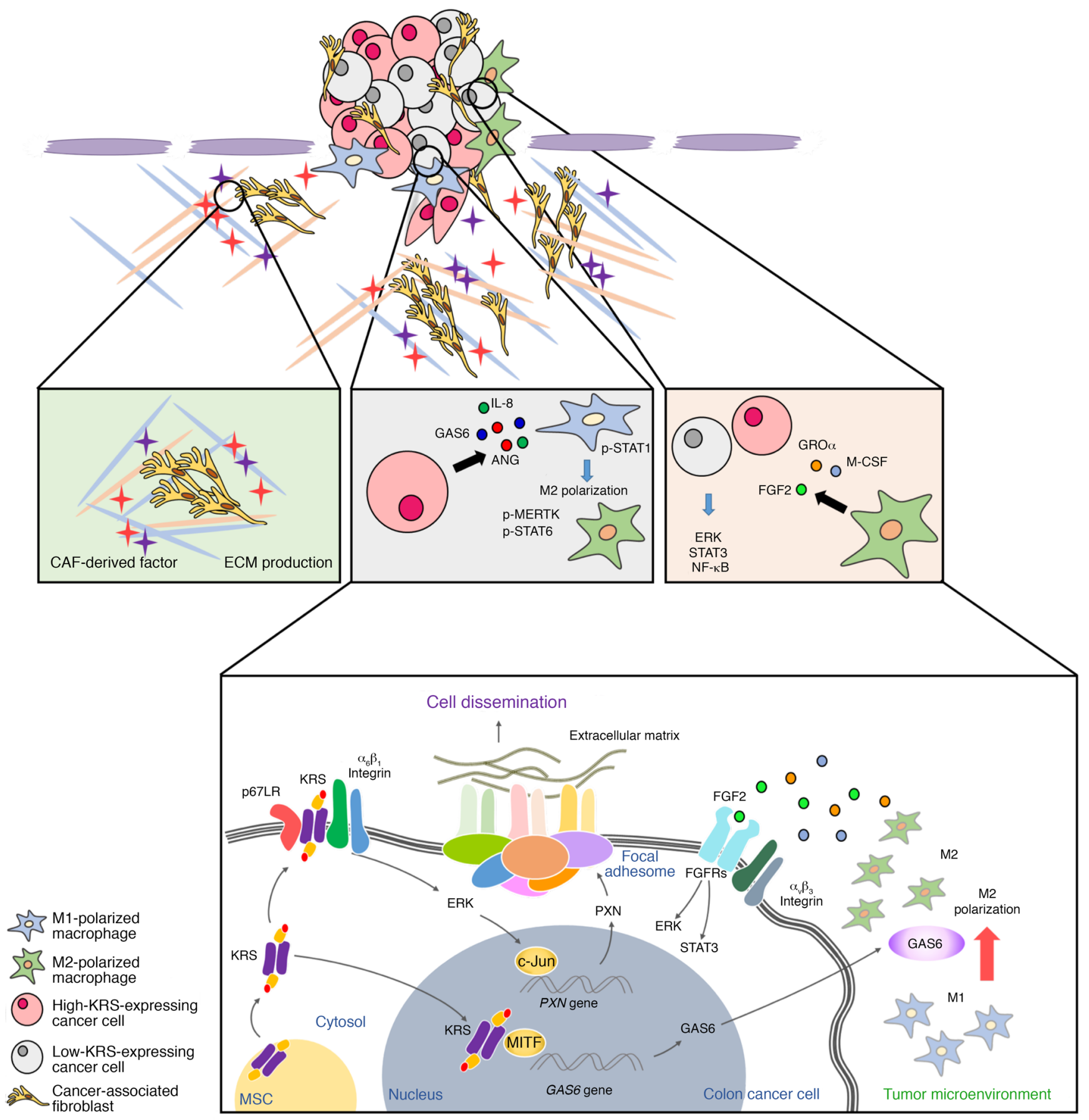

Figure 12. A schematic diagram for the disseminative phenomenon that relies on microenvironmental remodeling through a positive-feedback loop between KRS-positive colon cancer cells and environmental M2 macrophages for KRS-dependent metastasis. KRS-positive cancer spheroids induce GAS6/IL-8/ANG for M2 macrophage polarization, and then M2 macrophages secrete FGF2/GRO $\alpha / \mathrm{M}$-CSF for signaling activation in cancer spheroids for invasive outgrowth or dissemination. MSC, multi-tRNA synthetase complex.

rophages using $100 \mathrm{ng} / \mathrm{ml}$ lipopolysaccharide and $20 \mathrm{ng} / \mathrm{ml} \mathrm{IFN}-\gamma$ for 72 hours or alternatively differentiated into M2 macrophages using 20 $\mathrm{ng} / \mathrm{ml} \mathrm{IL}-4$ and $20 \mathrm{ng} / \mathrm{ml} \mathrm{IL-13}$ for 72 hours. Normal colon CCD-18Co fibroblasts were obtained from ATCC and cultured according to the standard protocols. Human monocytes from the peripheral blood of healthy donors were kindly provided by Chungnam National University Hospital (Daejeon, Republic of Korea). All cells were acquired or purchased with confirmations for the genotypic authentications from suppliers, and regularly tested for mycoplasma contamination.

Animals. BALB/c and C57BL/6 mice were purchased from Orient Bio (Seungnam, Republic of Korea). Chemical-induced colorectal cancer in mice was induced following a previously described protocol (36) with slight modifications. In brief, azoxymethane (AOM; 10 $\mathrm{mg} / \mathrm{kg}$; Sigma-Aldrich) was administered i.p. to 7-week-old BALB/c 
(control, $n=4$; AOM-DSS model, $n=15$ ) or C57BL/6 male mice (WT, $n=4 ; \mathrm{Krs}^{-/+}$heterozygous $\left.\mathrm{KO}, \mathrm{n}=4\right)$ to cause colon carcinogenesis. Mice were then treated with 3 cycles of $2.5 \%$ dextran sodium sulfate (DSS; Sigma-Aldrich) in drinking water. Each cycle consisted of 1 week of DSS-water followed by 2 weeks of tap water as drinking water. The mice were then sacrificed 10 weeks after the AOM administration. Tail vein injection experiments were performed with 8-week-old female BALB/c mice ( $n=5$ per group). CT2 6 cells $\left(10^{5}\right.$ cells per 200 $\mu \mathrm{l}$ in sterile $\mathrm{PBS}$ ) were injected into mouse tail veins using a 25 -gauge needle. At day 21 , the mice were sacrificed, and peritoneal tumors were histologically examined. WT or $\mathrm{Krs}^{-/+}$heterozygous KO mice (C57BL/6, 10-week-old males, $n=5$; ref. 12) were used for immunohistochemical analysis.

Colon orthotopic transplantation and metastasis analysis. Colon orthotopic transplantation of CT26 cells (CT26-shControl and CT26KRS-suppressed cells: CT26-shKRS\#2 and CT26-shKRS\#5) was performed as explained previously (27). Both CT26 cell lines were first stably infected with retroviral pMSCV-Luc2 vector (37), and then injected s.c. into BALB/c-nude (female, 6-week-old; Orient Bio) mice. Eight weeks later, cells were prepared from the syngeneic tumors. Then, $10^{5}$ cells mixed with Matrigel were orthotopically transplanted to the colon ( $n=4$ for each cell line), and luciferase signals from the primary and metastatic tumors were measured weekly using an IVIS in vivo animal imaging system (PerkinElmer) following i.p. injection of luciferin (122796, PerkinElmer). For inhibitor treatments, YH16899 (12) at $100 \mathrm{mg} / \mathrm{kg}(n=4)$ or the specific MerTK inhibitor RXDX-106 (S8570, Selleck Chemicals) at $30 \mathrm{mg} / \mathrm{kg}(n=4)$ was injected i.p. every other day 1 week after transplantation of CT26-shControl cells. Three weeks after transplantation, luciferase signal analysis of animals was done before and after laparotomy.

Spheroid formation and embedding into 3D collagen I gels. Spheroids of HCT116, SW620, and CT26 cells with modulated KRS expression levels were prepared and manipulated for time-lapse imaging and Western blot analysis, as previously described (12).

Extract preparation from the spheroids or $2 \mathrm{D}$ macrophages and Western blots. Colon cancer spheroids in 3D collagen I gels were washed with ice-cold PBS and then homogenized with truncated pipette tips (3 times for 20 minutes on ice) in modified RIPA buffer (50 mM Tris-HCl, 150 $\mathrm{mM} \mathrm{NaCl}, 1 \% \mathrm{NP}-40$, and $0.25 \%$ sodium deoxycholate) with a protease inhibitor cocktail (GenDepot). The spheroid extracts or whole cell extracts of macrophages in 2D cultures were processed before standard Western blotting, as described previously (12). The primary antibodies used were as follows: $\alpha$-SMA (A2547, mouse mAb) from Sigma-Aldrich; Hsp90 (4875, rabbit polyclonal [pAb]), MerTK (4319s, rabbit pAb), pY ${ }^{701}$ STAT1 (9167s, rabbit pAb), pY ${ }^{705}$ STAT3 (9145s, rabbit pAb), STAT6 (9362s, rabbit pAb), pY Y41STAT6 (9361s, rabbit pAb), ERKs (9102s, rabbit pAb), phospho-ERKs (4376s, rabbit pAb), and pY ${ }^{653 / 654}$ FGFR (3471s, rabbit pAb) from Cell Signaling Technology; paxillin (610569, mouse $\mathrm{mAb}$ ) from BD Biosciences; $\mathrm{pY}^{749 / 753 / 754}$ MerTK (ab14921, rabbit pAb) and laminin receptor (ab133645, rabbit $\mathrm{pAb}$ ) from Abcam; $\mathrm{pY}^{118}$ paxillin (sc-101774, rabbit pAb), STAT1 (sc-346, rabbit pAb), STAT3 (sc-482, rabbit pAb), FGFR1 (sc-121, rabbit pAb), FGFR2 (sc-122, rabbit pAb), MMP9 (sc-21733, mouse mAb), histone H1 (sc-10806, rabbit pAb), and $\alpha$-tubulin (sc-5286, rabbit pAb) from Santa Cruz Biotechnology; GAS6 (AF986, AF885, goat pAb) and MMP7 (MAB9071, mouse mAb) from R\&D Systems; $\alpha_{6}$ integrin (CBL458, mouse mAb) from Merck; EGFR (PA1-1110, rabbit pAb) from Thermo Fisher Scientific; MMP1 (38) and membranous KRS (against N-terminal ${ }^{1}$ MAAVQAAEVK VDGSEPKLSK NELKRRLKAE KKVAEKEAKQ $K^{42}$ ) or full-length KRS from Neomics. See complete unedited blots in the supplemental material.

Time-lapse imaging of cells in 3D ECM gels. Various stable cell spheroids were embedded in 3D collagen I gels ( $2 \mathrm{mg} / \mathrm{ml}$; BD Biosciences), and time-lapse images were collected for the indicated periods using an IX81-ZDC microscope (Olympus). The images were saved for the indicated times and presented in a $0^{\text {day }}: 00^{\text {hour}}: 00^{\text {minute }}$ format in the snap images. The microscope was equipped with a 10 -well Chamlide Incubator system (Live Cell Instrument), and an environmental chamber mounted on the microscope maintained constant conditions of $37^{\circ} \mathrm{C}, 5 \% \mathrm{CO}_{2}$, and $95 \%$ humidity. Spheroid images included yellow fractions to depict the phenotypes (numerator) out of total spheroids (denominator) analyzed.

Indirect immunofluorescence. Human normal colon CCD-18Co fibroblasts embedded in 3D collagen I gels $(2 \mathrm{mg} / \mathrm{ml}$; BD Biosciences) within polydimethylsiloxane (PDMS) were immunostained using antibodies against $\alpha$-SMA (Sigma-Aldrich) or laminins (Dako) and DAPI for the nucleus. Immunofluorescence images were acquired on a Nikon Eclipse Ti confocal laser scanning microscope with a Nikon Plan-Apochromat VC objective (20×/0.75 NA WD $1.00 \mathrm{~mm})$.

Chromatin immunoprecipitation. Chromatin immunoprecipitation (ChIP) assays were performed using the ChIP-IT Express kit (Active Motif), following the manufacturer's protocols and as described previously (12). The sheared chromatin from different spheroids was immunoprecipitated using MiTF (Abcam) or c-Jun (Cell Signaling Technology) antibodies and captured with protein $G$ magnetic beads (Bio-Rad). The isolated chromatin was analyzed by 36 cycles of PCR. Ten microliters of each PCR product was separated via $2 \%$ agarose electrophoresis.

Coculture in 3D collagen I gels. The Operetta/Harmony HCS Platform (PerkinElmer) was used to analyze the migration and invasion of cancer cells toward macrophages, and vice versa, in 3D collagen I gels. Monocytes or macrophages were embedded as a thin layer $(50 \mu \mathrm{l} /$ well in 24-well plates) at the bottom of the gels, and KRS-modulated HCT116 cancer cells were embedded as a monolayer on the upper side of the gels. In an opposite manner, KRS-modulated HCT116 cancer cells were embedded as a monolayer at the bottom of the gels, and monocytes or macrophages were embedded as a thin layer ( $50 \mu \mathrm{l} /$ well in 24 -well plates) on the upper side of the gels. Then the cancer cells or macrophages on the top of the gels were covered with additional gel (200 $\mu \mathrm{l})$. In some cases, treatment with vehicle, GAS6, GAS6 plus IL-8, or GAS6 plus IL-8 plus ANG was given to the M1 macrophage coculture with HCT116-shControl or HCT116-shKRS\#2 cells. HCT116 cancer cells were stained with Cell Tracker Green (CMFDA, Thermo Fisher Scientific), and macrophages were stained with Cell Tracker Red (CMTPX, Thermo Fisher Scientific). The location of cells in the gels was determined at days 5 and 10 after embedding. The confocal image acquisition was performed by the Operetta HCS System, which allowed robust and reliable cell counting within different planes of the matrix (at 10- or 30- $\mu$ m intervals). As a result, graphic presentation of the number of cells in each plane ( $y$ axis) and the relative migration distance ( $x$ axis) was presented.

Cytokine antibody array. A human cytokine antibody array (AAHCYT-3, RayBiotech) was used to measure the synthesis or secretion levels of 42 cytokines by HCT116 spheroids (shControl-expressing, KRS-suppressed, or KRS-overexpressing cells) in 3D collagen I gels for 24 hours. The CM were used for the array analysis. The Human 
XL Cytokine Array Kit (ARY022B, R\&D Systems), Multiplex Human Cytokine ELISA Kit (EM10002, ANOGEN), Arginase Activity Assay Kit (ab180877, Abcam), and Human GAS6 ELISA Kit (E-EL-H0078, Elabscience) were also used to simultaneously detect differences in cytokine secretion profiles in the CM of THP-1 or human macrophages (monocyte, M1-polarized, M2-polarized) cells, following the manufacturers' protocols. The relative expression levels of 102 human soluble cytokines were determined.

Collagen contraction analysis. Differentiated M1 macrophages were treated with GAS6 (10 ng/ml), IL-8 (50 ng/ml), and/or ANG (50 ng/ml) or CM from HCT116 cells with different KRS expression levels for 4 days. CCD-18Co cells mixed with $70 \mu \mathrm{l}$ collagen I solution $(2 \mathrm{mg} / \mathrm{ml})$ were overlaid on top of $50 \mu \mathrm{l}$ collagen I gel $(2 \mathrm{mg} / \mathrm{ml})$ per well. CM from the macrophages were then added to CCD-18Co cells $\left(10^{4}\right.$ cells per well) embedded in 3D collagen I gels for an additional 4 days, before imaging of the collagen contraction status, using phasecontrast microscopy (CKX41, Olympus).

Analysis of subcellular protein extracts. Cell fractionation was performed for the plasma membrane, cytosol, and nucleus fractions using a Subcellular Proteome Extraction Kit (Calbiochem), as indicated by the manufacturer. HCT116 shControl (transfected with control shRNA vector), HCT116 cells stably transfected with shKRS (targeting sequences of \#2 or \#5), Myc-KRS-overexpressing cells (KRS-WT), and other KRS mutants $(\Delta \mathrm{C} 5$, T52A, or S207A) were harvested for their membranal, nuclear, and cytosolic fractions. The cell fractions were normalized for standard Western blot analysis and probed using antibodies against the indicated molecules.

Immunohistochemistry. Dissected colon and lung tissues from the experimental animals were fixed and stored in $4 \%$ formaldehyde, and then processed, embedded in paraffin, and cut into $6-\mu \mathrm{m}$-thick sections. Human colon cancer or adjacent tissues were purchased from US Biomax. After deparaffinization, antigen retrieval was performed by boiling of the sections for 5 minutes in sodium citrate solution (0.01 $\mathrm{M}, \mathrm{pH}$ 6.0). Then, immunohistochemistry was performed using serial sections of animal tissues or paired normal or tumor human colon tissues, as previously described (12), using normal rabbit IgG (sc2027), normal mouse IgG (sc-2025), normal goat IgG (sc-2028, Santa Cruz Biotechnology), KRS (Neomics), CD206 (ab64693, rabbit pAb, Abcam), GAS6 (R\&D Systems), and CD11c (MA11C5, mAb, Thermo Fisher Scientific) antibodies. Images include scale bars at $100 \mu \mathrm{m}$.

Real-time PCR. Total RNA was extracted from cells in 10\% FBS-containing condition or in 3D collagen I gels, using QIAzol (Qiagen) according to the manufacturer's protocol. One microgram of total RNA was reverse-transcribed using the amfiRivert Platinum cDNA Synthesis master mix (GenDepot) and ReverTra Ace qPCR RT Master Mix with gDNA Remover (Toyobo). Primers were designed using Integrated DNA Technologies (IDT) software as follows: human ITGAM (CD11b) mRNA, forward 5'-CAGTGTGACATCCCGTTCTT-3' and reverse 5'-CACGATCAGGAGGTGGTTATG-3'; humanITGAX (CD11c) mRNA, forward5'-GTTAGCAGCCACGAACAATTC-3' and reverse 5'-TCCCTCTGTCCCAGGTTATT-3'; human TNFA mRNA, forward 5'-GATCCCTGACATCTGGAATCTG-3' and reverse 5'-GAAACATCTGGAGAGAGGAAGG-3'; human IL1B
mRNA, forward 5'-СТСТСАССТСТССТАСТСАСТT-3' and reverse 5'-TCAGAATGTGGGAGCGAATG-3'; human IL6 mRNA, forward 5'-ATAGGACTGGAGATGTCTGAGG-3' and reverse 5'-GCTTGTGGAGAAGGAGTTCATAG-3'; CD206, forward 5'-GGACGTGGCTGTGGATAAAT-3' and reverse 5'-ACCCAGAAGACGCATGTAAAG-3'; IL10, forward 5'-GCTGGAGGACTTTAAGGGTTAC-3' and reverse 5'-GATGTCTGGGTCTTGGTTCTC-3'; GAS6, forward 5'-TCTGTGGCACTGGTAGACTAT-3' and reverse 5'-CGCAGACCTTGATCTCCATTAG-3'; FN1, forward 5'-CCACAGTGGAGTATGTGGTTAG-3' and reverse 5'-CAGTCCTTTAGGGCGATCAAT-3'; human KRS (KARS) mRNA, forward 5'-GAGAAGGAGGCCAAACAGAA-3' and reverse 5'-CTCAGGACCCACACCATTATC-3'; human GAPDH mRNA, forward 5'-GGTGTGAACCATGAGAAGTATGA-3' and reverse 5'-GAGTCCTTCCACGATACCAAAG-3'; human LAMA1 mRNA, forward 5'-CGAGGCCTACCTTGGAAATAAG-3' and reverse 5'-AGGTTACTGTCTACCGTCTCTAC-3'; and human LAMB1 mRNA, forward 5'-CAGCAGCCGATGTGGTAATA-3' and reverse 5'-CCGTGTAGTTTGTTCCCTTCT-3'.

Statistics. Nonparametric analyses were conducted using a 1-way ANOVA with Dunnett tests for real-time PCR analysis. Otherwise, Student's $t$ test was performed for statistical comparisons of mean values to determine significance. A $P$ value less than 0.05 was considered significant.

Study approval. BALB/c and C57BL/6 mice were purchased from Orient Bio. Mice were housed in a specific pathogen-free room with controlled temperature and humidity. All animal care was in accordance with protocols approved by the Seoul National University Institutional Animal Care and Use Committee.

\section{Author contributions}

SHN performed most experiments. DK and DL helped with animal experiments. JWJ, HJK, NHK, and JEK helped with imaging experiments and with reagents. HML and EKJ helped with macrophages and protocols for their uses. DGS helped with 3D experiments. SK, and JWL reviewed the manuscript and discussed the data. JWL wrote the manuscript.

\section{Acknowledgments}

Kind help with operation of the Operetta/Harmony HCS Platform at a PerkinElmer core facility in the College of Pharmacy, Seoul National University, was given by PerkinElmerKorea. This work was supported by the Basic Science Research Program through the National Research Foundation of Korea funded by the Ministry of Science, ICT and Future Planning (NRF-2018M3A9C8020027 and NRF-2017R1A2B3005015), the Tumor Microenvironment Globarl Core Research Center (20110030001), and the Medicinal Bioconvergence Research Center (NRF-2013M3A6A4044019) to JWL.

Address correspondence to: Jung Weon Lee, College of Pharmacy, Seoul National University, 1 Gwanak-ro, Gwanak-gu, Seoul 08826, Republic of Korea. Phone: 8228802495; Email: jwl@snu.ac.kr.
1. Meng F, Wu G. The rejuvenated scenario of epithelial-mesenchymal transition (EMT) and cancer metastasis. Cancer Metastasis Rev. 2012;31(3-4):455-467.
2. Thiery JP, Lim CT. Tumor dissemination: an EMT affair. Cancer Cell. 2013;23(3):272-273.

3. Smith HA, Kang Y. The metastasis-promoting roles of tumor-associated immune cells. J Mol
Med. 2013;91(4):411-429.

4. Mikuła-Pietrasik J, Uruski P, Tykarski A, Książek K. The peritoneal "soil" for a cancerous "seed": a comprehensive review of the pathogenesis of 
intraperitoneal cancer metastases. Cell Mol Life Sci. 2018;75(3):509-525.

5. Kim S, You S, Hwang D. Aminoacyl-tRNA synthetases and tumorigenesis: more than housekeeping. Nat Rev Cancer. 2011;11(10):708-718.

6. Kim DG, et al. Interaction of two translational components, lysyl-tRNA synthetase and p40/37LRP, in plasma membrane promotes laminin-dependent cell migration. FASEB J. 2012;26(10):4142-4159.

7. Yannay-Cohen N, et al. LysRS serves as a key signaling molecule in the immune response by regulating gene expression. Mol Cell. 2009;34(5):603-611.

8. Young HJ, Lee JW, Kim S. Function of membranous lysyl-tRNA synthetase and its implication for tumorigenesis. Biochim Biophys Acta. 2016;1864(12):1707-1713.

9. Kim DG, et al. Chemical inhibition of prometastatic lysyl-tRNA synthetase-laminin receptor interaction. Nat Chem Biol. 2014;10(1):29-34.

10. Boulos S, et al. Serine 207 phosphorylated lysyl-tRNA synthetase predicts disease-free survival of non-small-cell lung carcinoma. Oncotarget. 2017;8(39):65186-65198.

11. Duchon AA, St Gelais C, Titkemeier N, Hatterschide J, Wu L, Musier-Forsyth K. HIV-1 exploits a dynamic multi-aminoacyl-tRNA synthetase complex to enhance viral replication. JVirol. 2017;91(21):e01240-17.

12. Nam SH, et al. Noncanonical roles of membranous lysyl-tRNA synthetase in transducing cell-substrate signaling for invasive dissemination of colon cancer spheroids in 3D collagen I gels. Oncotarget. 2015;6(25):21655-21674.

13. Kim SB, et al. Caspase- 8 controls the secretion of inflammatory lysyl-tRNA synthetase in exosomes from cancer cells. J Cell Biol. 2017;216(7):2201-2216.

14. Komohara Y, Takeya M. CAFs and TAMs: maestros of the tumour microenvironment. JPathol. 2017;241(3):313-315.

15. Wood SL, Pernemalm M, Crosbie PA, Whetton $\mathrm{AD}$. The role of the tumor-microenvironment in lung cancer-metastasis and its relationship to potential therapeutic targets. Cancer Treat Rev. 2014;40(4):558-566.

16. Taddei ML, Giannoni E, Comito G, Chiarugi P.
Microenvironment and tumor cell plasticity: an easy way out. Cancer Lett. 2013;341(1):80-96.

17. Dehne N, Mora J, Namgaladze D, Weigert A, Brüne B. Cancer cell and macrophage cross-talk in the tumor microenvironment. Curr Opin Pharmacol. 2017;35:12-19.

18. Goswami KK, Ghosh T, Ghosh S, Sarkar M, Bose A, Baral R. Tumor promoting role of anti-tumor macrophages in tumor microenvironment. Cell Immunol. 2017;316:1-10.

19. Attieh Y, Vignjevic DM. The hallmarks of CAFs in cancer invasion. Eur J Cell Biol. 2016;95(11):493-502.

20. Mori S, Takada Y. Crosstalk between Fibroblast Growth Factor (FGF) receptor and integrin through direct integrin binding to FGF and resulting integrin-FGF-FGFR ternary complex formation. Med Sci. 2013;1(1):20-36.

21. Wang N, Liang H, Zen K. Molecular mechanisms that influence the macrophage $\mathrm{m} 1-\mathrm{m} 2$ polarization balance. Front Immunol. 2014;5:614.

22. Chiu KC, et al. Polarization of tumorassociated macrophages and Gas6/Axl signaling in oral squamous cell carcinoma. Oral Oncol. 2015;51(7):683-689.

23. Crittenden MR, et al. Mertk on tumor macrophages is a therapeutic target to prevent tumor recurrence following radiation therapy. Oncotarget. 2016;7(48):78653-78666.

24. Sica A, Mantovani A. Macrophage plasticity and polarization: in vivo veritas. J Clin Invest. 2012;122(3):787-795.

25. Dransfield I, Farnworth S. Axl and Mer receptor tyrosine kinases: distinct and nonoverlapping roles in inflammation and cancer. In: Gregory $\mathrm{CD}$, ed. Apoptosis in Cancer Pathogenesis and Anti-cancer Therapy: New Perspectives and Opportunities. Cham, Switzerland: Springer International Publishing; 2016:113-132.

26. Ofir-Birin Y, et al. Structural switch of lysyl-tRNA synthetase between translation and transcription. Mol Cell. 2013;49(1):30-42.

27. Fumagalli A, et al. A surgical orthotopic organoid transplantation approach in mice to visualize and study colorectal cancer progression. Nat Protoc. 2018;13(2):235-247.

28. Yamaguchi H, Sakai R. Direct interaction between carcinoma cells and cancer associated fibroblasts for the regulation of cancer invasion. Cancers (Basel). 2015;7(4):2054-2062.

29. Guo F, Cen S, Niu M, Javanbakht H, Kleiman L. Specific inhibition of the synthesis of human lysyl-tRNA synthetase results in decreases in tRNA(Lys) incorporation, tRNA(3) (Lys) annealing to viral RNA, and viral infectivity in human immunodeficiency virus type 1.J Virol. 2003;77(18):9817-9822.

30. Qin Y, Rodin S, Simonson OE, Hollande F. Laminins and cancer stem cells: partners in crime? Semin Cancer Biol. 2017;45:3-12.

31. Park SG, et al. Human lysyl-tRNA synthetase is secreted to trigger proinflammatory response. Proc Natl Acad Sci U S A. 2005;102(18):6356-6361.

32. Wallet MA, et al. MerTK is required for apoptotic cell-induced T cell tolerance. J Exp Med. 2008;205(1):219-232.

33. Freire-de-Lima CG, Xiao YQ, Gardai SJ, Bratton DL, Schiemann WP, Henson PM. Apoptotic cells, through transforming growth factor-beta, coordinately induce anti-inflammatory and suppress pro-inflammatory eicosanoid and NO synthesis in murine macrophages. J Biol Chem. 2006;281(50):38376-38384.

34. Graham DK, DeRyckere D, Davies KD, Earp HS. The TAM family: phosphatidylserine sensing receptor tyrosine kinases gone awry in cancer. Nat Rev Cancer. 2014;14(12):769-785.

35. Bronte V, Murray PJ. Understanding local macrophage phenotypes in disease: modulating macrophage function to treat cancer. Nat Med. 2015;21(2):117-119.

36. Neufert C, Becker C, Neurath MF. An inducible mouse model of colon carcinogenesis for the analysis of sporadic and inflammation-driven tumor progression. Nat Protoc. 2007;2(8):1998-2004.

37. Lee D, et al. Interaction of tetraspan(in) TM4SF5 with CD44 promotes self-renewal and circulating capacities of hepatocarcinoma cells. Hepatology. 2015;61(6):1978-1997.

38. Kim MS, Kim YK, Cho KH, Chung JH. Regulation of type I procollagen and MMP-1 expression after single or repeated exposure to infrared radiation in human skin. Mech Ageing Dev. 2006;127(12):875-882. 